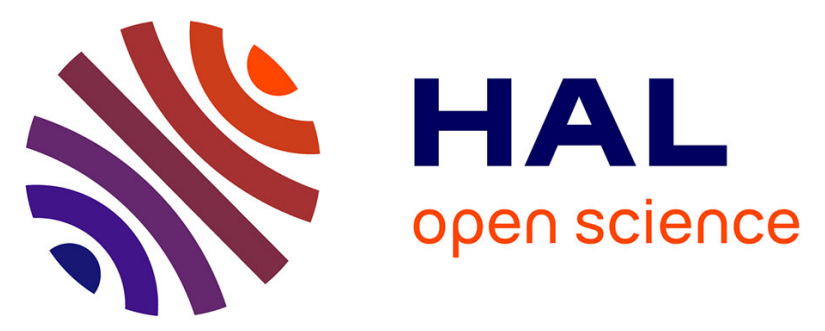

\title{
Mesoscopic modeling of spacing and grain selection in columnar dendritic solidification: Envelope versus phase-field model
}

Alexandre Viardin, Miha Založnik, Youssef Souhar, Markus Apel, Hervé Combeau

\section{To cite this version:}

Alexandre Viardin, Miha Založnik, Youssef Souhar, Markus Apel, Hervé Combeau. Mesoscopic modeling of spacing and grain selection in columnar dendritic solidification: Envelope versus phase-field model. Acta Materialia, 2017, 122, pp.386 - 399. 10.1016/j.actamat.2016.10.004 . hal-01709176

\section{HAL Id: hal-01709176 \\ https://hal.univ-lorraine.fr/hal-01709176}

Submitted on 14 Feb 2018

HAL is a multi-disciplinary open access archive for the deposit and dissemination of scientific research documents, whether they are published or not. The documents may come from teaching and research institutions in France or abroad, or from public or private research centers.
L'archive ouverte pluridisciplinaire HAL, est destinée au dépôt et à la diffusion de documents scientifiques de niveau recherche, publiés ou non, émanant des établissements d'enseignement et de recherche français ou étrangers, des laboratoires publics ou privés. 


\title{
Mesoscopic modeling of spacing and grain selection in columnar dendritic solidification: envelope versus phase-field modele ${ }^{\text {th }}$
}

\author{
Alexandre Viardin $^{\mathrm{a}}$, Miha Založnik ${ }^{\mathrm{b}}$, Youssef Souhar ${ }^{\mathrm{b}}$, Markus Apel $^{\mathrm{a}}$, Hervé Combeau ${ }^{\mathrm{b}}$ \\ ${ }^{a}$ Access e.V., Intzestr. 5, D-52072 Aachen, Germany \\ ${ }^{b}$ Institut Jean Lamour, CNRS - Université de Lorraine, F-54011 Nancy CEDEX, France
}

\begin{abstract}
We investigate and assess the capability of the mesoscopic envelope model of dendritic solidification to represent the growth of columnar dendritic structures. This is done by quantitative comparisons to phase-field simulations in two dimensions. While the phase-field model resolves the detailed growth morphology at the microscale, the mesoscopic envelope model describes a dendritic grain by its envelope. The envelope growth velocities are calculated by an analytical dendrite-tip model and matched to the numerical solution of the solute concentration field in the vicinity of the envelope. The simplified representation of the dendrites drastically reduces the calculation time compared to phase field. Larger ensembles of grains can therefore be simulated. We show that the mesoscopic envelope model accurately reproduces the evolution of the primary branch structure, the undercooling of the dendrite tips, and the solidification path in the columnar mushy zone. We further show that it can also correctly describe the transient adjustments of primary spacing, both by spacing increase due to elimination of primary branches and by spacing reduction due to tertiary rebranching. Elimination and tertiary rebranching are also critical phenomena for the evolution of grain boundaries between columnar grains that have a different crystallographic orientation with respect to the temperature gradient. We show that the mesoscopic model can reproduce the macroscopic evolution of such grain boundaries for small and moderate misorientation angles, i.e., up to $30^{\circ}$. It is therefore suitable for predicting the texture of polycrystalline columnar structures. We also provide guidelines for the calibration of the main parameters of the mesoscopic model, required to obtain reliable predictions.
\end{abstract}

Keywords: Alloy solidification, Dendritic growth, Multiscale modeling, Model validation, Microstructure selection, Directional solidification, Phase-field method

\section{Introduction}

Dendritic crystal grains are the most common growth morphology in solidification of metallic alloys. Dendritic growth depends on the interplay between diffusion of heat and solute, capillarity, and fluid flow; phenomena that span a wide range of length and time scales. In polycrystalline materials the growth is also influenced by collective interactions. The grains influence each other due to the overlap of thermal and solutal fields surrounding each growing grain. These interactions are decisive for the development of features of the grain structure that determine the properties of a polycrystalline material: the size and the shape of the grains, the spacings of the dendritic ramifications, and the crystallographic texture [38].

To be able to study the growth of such patterns, a multiscale description is required, ranging from the dendrite tip

\footnotetext{
This is a post-peer-review, pre-copyedit version of an article published in Acta Materialia: A. Viardin, M. Založnik, Y. Souhar, M. Apel, H. Combeau, Mesoscopic modeling of spacing and grain selection in columnar dendritic solidification: Envelope versus phase-field model, Acta Mater. 122 (2017) 386-399. The final authenticated version is available online at: http:// $\mathrm{dx}$.doi.org/10.1016/j. actamat.2016.10.004.

Email address: a.viardin@access-technology.de (Alexandre Viardin)
}

to the collective scale. Phase-field methods can describe the detail of the dendritic structure. Due to the high computational cost, their scale of application is usually limited to a few grains and to two dimensions. Large ensembles of grains in $3 \mathrm{D}$ can be simulated, but such computations require complex high-performance parallel computing algorithms and massive supercomputing resources $[14,41]$. To overcome the limitations of phase-field methods, i.e., to allow simulation on larger scales or with larger arrays of dendrites at acceptable cost, several mesoscopic approaches have been proposed. Some models keep the notion of the solid-liquid interface in their description of the solidification microstructures and propose to obtain a higher efficiency by a coarser, less accurate description of the interface and its kinetics [8, 18, 47]. Other mesoscopic models are based on simplified representations of the microstructure. The dendrite-needle-network (DNN) model [42, 43] represents the dendritic network of primary, secondary and higher order branches by a network of sharp needles whose dynamics can be prescribed without resolving the solid-liquid interface. Several models are based on the notion of a dendrite envelope, a smooth surface connecting all actively growing dendrite tips. The growth of the envelope is deduced from the velocities of the dendrite tips, 
calculated from the local undercooling by an analytical tip model. The CAFE [12, 28] and the front-tracking [4, 27] envelope-based models can be used up to the size of small castings, but they are not able to predict accurate grain shapes and rely on very approximate relations for modeling grain interactions.

Another approach to mesoscopic simulation of dendritic growth is the envelope model developed by Steinbach, Beckermann and coworkers [6, 32, 33]. In this model the dendrite envelope is calculated by an analytical LGK-type tip model that is matched to the concentration fields in the rigorously defined stagnant film around the envelope. The intra-grain solidification is described by a solid fraction field. The advantage of this model is that it predicts very accurate grain shapes and grain interactions. Extensive validations have shown that it gives physically realistic results for equiaxed dendritic growth $[30,32,33]$ in three dimensions. The first application of the mesoscopic envelope model to columnar growth has been developed by Delaleau et al. [6]. Using an arrangement of columnar grains identical to the one observed in an in situ directional solidification experiment, the structure and the growth dynamics of a few primary dendrite branches have been successfully reproduced.

A truly predictive modeling of columnar growth must describe the principal phenomena that govern the formation of the internal structure of a grain (primary dendrite arm spacing) and the competition between growing grains in a polycrystal. The primary spacing is a result of transient adjustments: spacing increase due to elimination of primary branches and spacing reduction due to creation of new primary branches by tertiary rebranching. In a polycrystal, elimination and tertiary rebranching at grain boundaries are also decisive for the competition between columnar dendrites with different crystallographic orientations [13, 44, 53].

In this paper we show that the mesoscopic envelope model correctly describes most of these phenomena and can thus be a powerful tool for studying columnar dendritic solidification. We investigate many aspects of the predicted columnar structure and we quantitatively compare them to twodimensional phase-field simulations that are used as a reference. These comparisons show that the mesoscopic model accurately reproduces the evolution of the primary branch structure, the undercooling of the dendrite tips, and the solidification path in the columnar mushy zone. We further show that it can correctly describe the transient adjustments of primary spacing, both by branch elimination and by tertiary rebranching. These phenomena are also reproduced at grain boundaries between columnar grains that have different crystallographic orientations with respect to the temperature gradient. We show that the mesoscopic model can reproduce the macroscopic evolution of such grain boundaries for small and moderate misorientation angles. It can therefore be suitable for predicting the growth competition in polycrystalline columnar structures. The influence of the main model parameter, the stagnant-film thickness, is carefully examined in all simulated configurations. We provide guidelines for the calibration of the the stagnant-film thickness, required to obtain reliable predictions.

\subsection{Mesoscopic envelope model}

The core idea of the mesoscopic envelope model is the description of a dendritic grain by its envelope - a virtual smooth surface that links the tips of the actively growing dendrite branches. The growth velocity of the envelope can thus be calculated from the velocities of the dendrite tips. The growth of the dendrite tips is controlled by the solute flux that they eject into their surroundings and is therefore determined by the local supersaturation of the liquid in the vicinity of the envelope. The branched dendritic structure inside the envelope is only implied and its details are not resolved; the interior of the envelope is instead described in a volume-averaged sense by a phase-fraction field. The phase solute concentrations are also described in a volumeaveraged sense. The phase change that determines the evolution of the structure, i.e., of the phase fraction field, inside the growing envelope is controlled by the exchange of solute with the surroundings of the grain. The transport of solute at the mesoscopic scale is described by volume-averaged transport equations.

The local growth speed of the envelope is given by the kinetics of the dendrite tips behind it. Their growth Péclet number, $\mathrm{Pe}_{\text {tip }}$, is obtained from the stagnant-film formulation of the Ivantsov solution for a steady-state parabolic tip that is written in terms of the supersaturation at a finite distance $\delta$ from the tip [5], $\Omega_{\delta}$ :

$$
\begin{aligned}
\Omega_{\delta}=\sqrt{\pi \mathrm{Pe}_{\text {tip }}} \exp \left(\mathrm{Pe}_{\text {tip }}\right) & {\left[\operatorname{erfc}\left(\sqrt{\mathrm{Pe}_{\text {tip }}}\right)\right.} \\
& \left.-\operatorname{erfc}\left(\sqrt{\mathrm{Pe}_{\text {tip }}\left[1+\frac{2 \delta}{R_{\text {tip }}}\right]}\right)\right]
\end{aligned}
$$

The supersaturation $\Omega_{\delta}$ is obtained from the numerically resolved concentration field in the liquid around the grain envelope. This corresponds to a matching of the locally valid tip solution to the solution of the mesoscopically valid solute transport equation at a distance $\delta$ from the envelope. In this way the tip kinetics and the mesoscale solutal interactions are fully coupled without resolving the structure of individual dendrite branches.

Eq. (1) is supplemented by the tip selection criterion, which can be written as $R_{\text {tip }}^{2} V_{\text {tip }}=d_{0} D_{1} / \sigma^{*}$, where $d_{0}=$ $\Gamma /\left(m_{\mathrm{L}} C_{1}^{*}(k-1)\right)$ is the solutal capillary length, $D_{1}$ is the liquid diffusion coefficient, $\sigma^{*}$ is the selection constant, $\Gamma$ is the Gibbs-Thomson coefficient, $m_{\mathrm{L}}$ is the liquidus slope, $C_{1}^{*}$ is the liquid concentration at the tip, and $k$ is the equilibrium partition coefficient. Then, the tip speed is given by

$$
V_{\text {tip }}=\frac{4 \sigma^{*} D_{1} \mathrm{Pe}_{\text {tip }}^{2}}{d_{0}} .
$$

Eqs. (1) and (2) together give the local tip speed at any point on the grain envelope. The stagnant-film thickness, $\delta$, is a model parameter.

The tips are assumed to grow in prescribed preferential growth directions. For example, a typical cubic crystal dendrite is approximated by six possible growth directions that 
are perpendicular to each other. The normal envelope growth velocity, $\vec{v}_{n}$, is then calculated from the local tip speed, $V_{\text {tip }}$, by the relation

$$
\vec{v}_{n}=V_{\text {tip }} \vec{n} \cos \theta
$$

where $\theta$ is the angle between the outward drawn normal to the envelope, $\vec{n}$, and the preferential growth direction that forms the smallest angle with the local envelope normal.

As in previous work [6, 30, 32, 33], the phase-field sharpinterface capturing method [36] is used to propagate the envelope on a numerical mesh. The phase-field equation for the propagation of the indicator function $\phi_{i}$ is [37]

$$
\begin{aligned}
\frac{\partial \phi_{i}}{\partial t}+v_{n} \vec{n} & \cdot \nabla \phi_{i}= \\
-b\left[\nabla^{2} \phi_{i}-\frac{\phi_{i}\left(1-\phi_{i}\right)\left(1-2 \phi_{i}\right)}{W^{2}}\right. & \left.-\left|\nabla \phi_{i}\right| \nabla \cdot\left(\frac{\nabla \phi_{i}}{\left|\nabla \phi_{i}\right|}\right)\right] .
\end{aligned}
$$

The parameters $W$ and $b$ of the method, as well as the grid size and timestep were selected following the guidelines determined by Souhar et al. [30]. To simulate polycrystals, each crystal orientation is described by its own indicator field $\phi_{i}$ that has its own set of preferential growth directions. This is not optimal with respect to the computation time but it is a very simple and robust way to account for multiple orientations.

The solute transport at the mesoscopic scale is described by volume averaged equations that are valid in the whole domain, i.e., both inside and outside the envelopes. The solid and liquid phases are assumed to be stationary. Solidification inside the envelope is modeled using the Scheil assumptions: thermodynamic equilibrium at the solid-liquid interface, negligible diffusion in the solid and instantaneous diffusion in the liquid. This implies that the concentration of the binary liquid inside the envelope is linked to the temperature field by $C_{1}^{*}=\left(T-T_{\mathrm{f}}\right) / m_{\mathrm{L}}$, where $C_{1}^{*}$ is the liquid equilibrium concentration, $T$ is the temperature, and $T_{\mathrm{f}}$ is the melting temperature of the pure solvent. These assumptions lead to the conservation equation for the solute in the liquid phase:

$$
g_{1} \frac{\partial C_{l}}{\partial t}=D_{1} \nabla \cdot\left(g_{1} \nabla C_{l}\right)+C_{l}(k-1) \frac{\partial g_{1}}{\partial t} .
$$

Outside the envelope, the material is fully liquid $\left(g_{1}=1\right)$ and Eq. (5) reduces to a single phase diffusion equation. Inside the envelope, the liquid is in thermodynamic equilibrium, such that $C_{l}=\left(T-T_{\mathrm{f}}\right) / m_{\mathrm{L}}$, where the temperature is assumed to be known. With $C_{1}$ known, Eq. (5) gives the evolution of the liquid fraction inside the envelope. The concentration of the solid, $C_{s}$, inside the envelope is given by

$$
\frac{\partial\left(g_{s} C_{s}\right)}{\partial t}=-k C_{1} \frac{\partial g_{1}}{\partial t}
$$

where $g_{\mathrm{s}}=1-g_{1}$ is the solid fraction. The average local concentration is defined as $\bar{C}=g_{1} C_{1}+\left(1-g_{1}\right) C_{\mathrm{s}}$.

\subsection{Phase-field model}

The phase-field model used is based on the multiphase field model [11, 34]. Its application to directional solidification was already described in [3, 10, 31]. Its calibration has been performed to match analytical solutions from [20] concerning the tip radius and the undercooling at steady state. A finite-difference correction [9] was used to improve the accuracy of the results.

\subsection{Simulation parameters}

We chose a succinonitrile-2 at.\%acetone alloy for our investigations. Its physical properties are given in Table 1 . Directional solidification was modeled by imposing a fixed temperature gradient in the vertical $(y)$ direction, $G_{T}=$ $2 \cdot 10^{4} \mathrm{~K} / \mathrm{m}$, and a cooling rate, $\dot{T}=-1.16 \mathrm{~K} / \mathrm{s}$, corresponding to a pulling speed of $V_{\text {pull }}=58 \mu \mathrm{m} / \mathrm{s}$ (Bridgman configuration). The simulations were performed in rectangular domains of $500 \mu \mathrm{m}$ height and widths between $250 \mu \mathrm{m}$ and $1000 \mu \mathrm{m}$. A so-called moving frame was used, i.e., a computational domain that moves with the dendrite tips, maintaining a minimum distance of $100 \mu \mathrm{m}$, which is around 4.5 times the diffusion length, $D_{1} / V_{\text {pull }}$, between the most advanced tip and the top boundary of the domain. This is sufficiently far to avoid confinement of the diffusion field at the top boundary. At the lateral vertical boundaries periodic boundary conditions were used in the phase-field model and symmetry boundary conditions in the mesoscopic model. Despite the difference in the boundary condition type the two configurations are equivalent as long as the solutions at the periodic boundaries remain symmetric. A fixed concentration of $C_{0}=2$ at.\%, a zero phase-field variable and envelope indicator functions $\phi_{i}=0$ were imposed at the top boundary and a zero normal derivatives at the bottom boundary. The grid spacing was $0.5 \mu \mathrm{m}$ in the phase-field simulations and $2.5 \mu \mathrm{m}$ in the mesoscopic simulations.

The simulations were initiated by circular seeds inserted at the bottom of the calculation domain. at an initial supersaturation of $\Omega_{\text {ini }}=0.20$. The steady-state supersaturation of the tips is around $\Omega=0.35$.

Where not stated otherwise, the stagnant-film thickness for the mesoscopic model was $\delta_{c}=1.18 l_{\text {diff }}{ }^{1}$, where $l_{\text {diff }}=D_{1} / V_{\text {pull }}=2.2 \cdot 10^{-5} \mathrm{~m}$ is the diffusion length at the tips. This follows the guidelines of Souhar et al. [30], who have shown that $\delta_{c} \sim l_{\text {diff }}$ gives the most accurate results for equiaxed growth. The tip selection parameter $\sigma^{*}$ for the mesoscopic model was determined from the steady-state tip radius obtained by the phase field model, $R_{\text {tip,PF }}=2.63 \mu \mathrm{m}$, using the tip selection criterion $R_{\text {tip,PF }}^{2} V_{\text {pull }}=\left(d_{0} D_{1}\right) / \sigma^{*}$. The tip velocity is $V_{\text {pull }}$ and the liquid concentration at the tip used in the capillary length was estimated from the supersaturation of a free

\footnotetext{
${ }^{1}$ This value was picked for practical reasons. Historically, the code that we used uses the ratio $\delta_{c} / R_{\mathrm{IV}}$ as input, where $R_{\mathrm{Iv}}$ is the theoretical freetip radius at the given tip speed. In the present case $R_{\mathrm{Iv}}=2.63 \mu \mathrm{m}$ and $\delta_{c}=10 R_{\mathrm{IV}}=1.18 D_{1} / V_{\text {pull }}$.
} 
Table 1

Parameters of the simulations

\begin{tabular}{ll} 
Thermophysical properties of the $S C N$-acetone alloy \\
Acetone concentration $\left(C_{0}\right)$ & 2.0 at. $\%$ \\
Melting temp. of pure $\mathrm{SCN}\left(T_{\mathrm{f}}\right)$ & $331.231 \mathrm{~K}$ \\
Liquidus slope $\left(m_{\mathrm{L}}\right)$ & $-2.45 \mathrm{~K} / \mathrm{at} \%$ \\
Partition coefficient $(k)$ & 0.103 \\
Diffusion coefficient in liquid $\left(D_{1}\right)$ & $1.27 \cdot 10^{-9} \mathrm{~m}^{2} / \mathrm{s}$ \\
Gibbs-Thomson coefficient $(\Gamma)$ & $6.525 \cdot 10^{-8} \mathrm{Km}$ \\
Interfacial energy $\left(\sigma_{\text {sl }}\right)$ & $6.525 \cdot 10^{-2} \mathrm{~J} / \mathrm{m}^{2}$ \\
Interfacial energy anisotropy & $0.93 \%$ \\
& \\
Solidification parameters & \\
Temperature gradient $\left(G_{T}\right)$ & $2 \cdot 10^{4} \mathrm{~K} / \mathrm{m}$ \\
Cooling rate $(T)$ & $-1.16 \mathrm{~K} / \mathrm{s}$ \\
(Pulling speed $\left.\left(V_{\text {pull }}\right)\right)$ & $5.8 \cdot 10^{-5} \mathrm{~m} / \mathrm{s}$ \\
Initial temperature at the & \\
bottom of the domain $\left(T_{\text {ini }}\right)$ & $325.2598 \mathrm{~K}$ \\
(Initial supersaturation at & \\
the bottom of the domain $\left.\left(\Omega_{\text {ini }}\right)\right)$ & 0.20 \\
& \\
Mesoscopic model parameters & \\
Tip selection constant $\left(\sigma^{*}\right)$ & 0.03282 \\
Stagnant-film thickness $\left(\delta_{c}\right)$ & $2.63 \cdot 10^{-5} \mathrm{~m}$ \\
\hline
\end{tabular}

tip, $\Omega_{\mathrm{Iv}}=\left(C_{1, \mathrm{IV}}^{*}-C_{0}\right) /\left((1-k) C_{1, \mathrm{Iv}}^{*}\right)$, given by the Ivantsov model: $\Omega_{\mathrm{Iv}}=\operatorname{Iv}\left(\mathrm{Pe}_{\mathrm{IV}}\right)=\sqrt{\pi \mathrm{Pe}_{\mathrm{IV}}} \exp \left(\mathrm{Pe}_{\mathrm{IV}}\right) \operatorname{erfc}\left(\sqrt{\mathrm{Pe}_{\mathrm{IV}}}\right)$, where $\mathrm{Pe}_{\mathrm{Iv}}=R_{\text {tip }, \mathrm{PF}} V_{\text {pull }} /\left(2 D_{1}\right)$. We obtained $\sigma^{*}=0.0328$.

We used the mesoscopic model code CrystalFOAM [30], developed on the OpenFOAM ${ }^{\circledR}$ [2] finite-volume platform. For the phase-field simulations, we used the software MICRESS [1].

\section{Mesoscopic representation of a columnar microstruc- ture}

An example of a mesoscopic simulation of transient columnar dendritic growth is shown in Fig. 1. The mesoscopic simulation is compared to a phase-field simulation, shown in the left part of each figure frame. The growth of the dendrites is initiated from two nuclei placed in the bottom corners of the domain of $250 \mu \mathrm{m}$ width. It starts with a fast growth of lateral dendrite arms horizontally along the bottom of the domain, where the supersaturation is the highest $(t=1 \mathrm{~s})$. Simultaneously, but at a slower rate, vertical dendrite arms grow along the temperature gradient. At $t=2 \mathrm{~s}$, when the lateral branches meet, the supersaturation is still the highest in the middle of the domain. This promotes the development of vertical secondary branches $(t=4 \mathrm{~s})$. In the mesoscopic model this is represented by a vertical expansion of the envelope. At $t=6 \mathrm{~s}$ we can see that only two of the secondary branches survive and develop into new primary branches. In the mesoscopic model this is manifested by the growth of the cusps at the end of the lateral wings of the envelope. The envelope between the new primary branches gradually merges together and the spacing is slightly adjusted $(t=8-48 \mathrm{~s})$. The steady state is reached at around $t=30 \mathrm{~s}$. The dendrite tips then grow at a constant speed of $58 \mu \mathrm{m} / \mathrm{s}$, equal to the pulling speed, and their undercooling is constant. The primary arms stabilize into a steady pattern with a slightly nonuniform but symmetric spacing. This is reproduced identically by both models.

We can see that the mesoscopic model represents the columnar structure by a "wavy" shape of the envelope and by a nonuniform solid-fraction field in the envelope. The patterns of the envelope shape and of the solid-fraction field match the primary branch structure of the columnar grains predicted by the phase-field model. We can also see that the concentration fields in the liquid ahead and between the primary branches very closely resemble that of the phase-field simulation. A more detailed representation is given by the plot of the vertical concentration profiles in Fig. 2, where we can see that the profiles and the tip concentrations match very well. We can also see that the solute concentration of the interacting tips of the columnar front is higher than that of a single free dendrite tip growing at the same speed in an infinite melt (Ivantsov). This indicates solutal interaction between the tips. The mesoscopic model captures such interactions with good accuracy. This can be confirmed by taking a closer look at the tip operating state. The concentrations at the dendrite tip are 2.926 and 2.945 at.\% for the phase-field and the mesoscopic model, respectively. This corresponds to tip undercoolings of 2.270 and $2.314 \mathrm{~K}$, respectively. The difference of $0.044 \mathrm{~K}$ is mostly due to the capillary undercooling that is not accounted for in the mesoscopic model. For a tip with a radius of $2.6 \mu \mathrm{m}$ the capillary undercooling is $2 \Gamma / R_{\text {tip }}=0.050 \mathrm{~K}$. In order to reveal the intensity of the solutal interactions between the primary tips, the concentration field in front of a freely growing tip is shown for comparison. It is given by the analytical Ivantsov solution for a parabolic tip growing in an infinite melt [17]. The supersaturation of the free tip is $\Omega_{\mathrm{IV}}=0.3363$, which corresponds to a concentration of 2.864 at.\%, and an undercooling of $2.116 \mathrm{~K}$. The difference of tip undercooling between both model solutions is much smaller than the difference between an interacting and a free tip. This shows that the mesoscopic model accurately represents the tip interactions.

Fig. 3 shows the comparison of vertical profiles of the average solid fraction from phase-field and mesoscopic models. They were obtained by averaging the solid fraction in the horizontal direction across the whole domain width, i.e., by calculating $\bar{g}_{s}(y)=\frac{1}{\left(x_{\max }-x_{\min }\right)} \int_{x_{\max }}^{x_{\max }} g_{s}(x, y) d x$ for each grid row. The corresponding full solid fraction maps for the phase-field (left) and the mesoscopic model (right) are shown in the inset. We can see that the mesoscopic simulation follows the phase-field solution closely. The fluctuations due to the presence of secondary arms are of course not reproduced by the mesoscopic model, where the solidfraction field inside the envelopes is smooth. The profiles are compared to a so-called closed-system Scheil law [21], i.e., a Scheil solidification path calculated for a constant average 


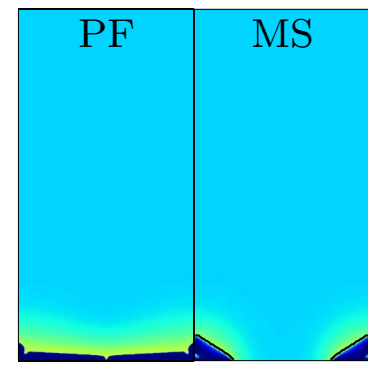

$1 \mathrm{~s}$

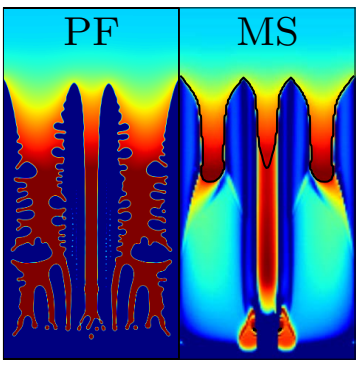

$8 \mathrm{~s}$

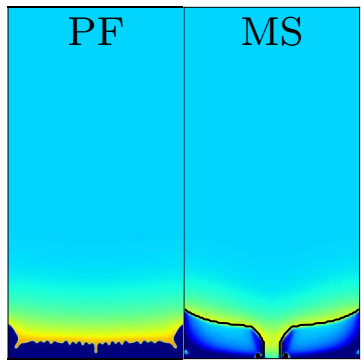

$2 \mathrm{~s}$

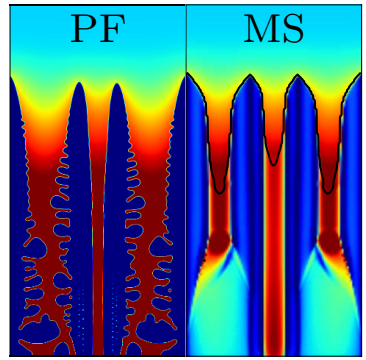

$10 \mathrm{~s}$

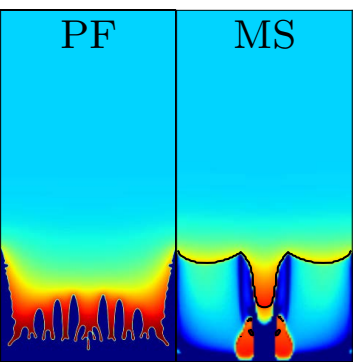

$4 \mathrm{~s}$

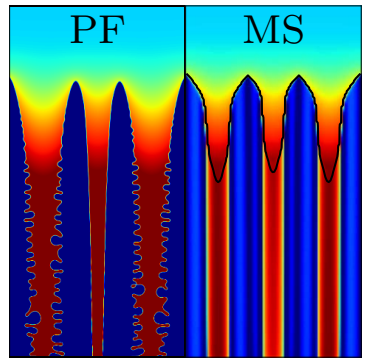

$15 \mathrm{~s}$

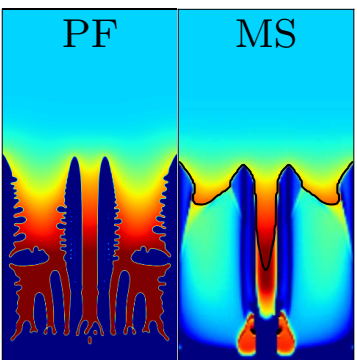

$6 \mathrm{~s}$

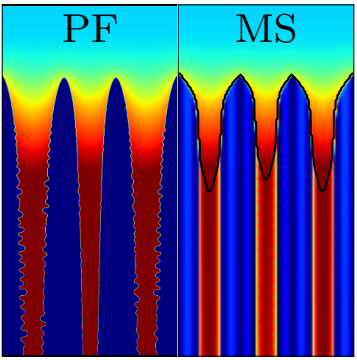

$48 \mathrm{~s}$

Fig. 1. The evolution of the columnar grains during directional solidification initiated from two nuclei placed in the bottom corners. Fields of average concentration calculated by phase field (PF, left) and by the mesoscopic (MS, right) model. The envelope of the mesoscopic model is shown as a black line. The domain width is $250 \mu \mathrm{m}$.

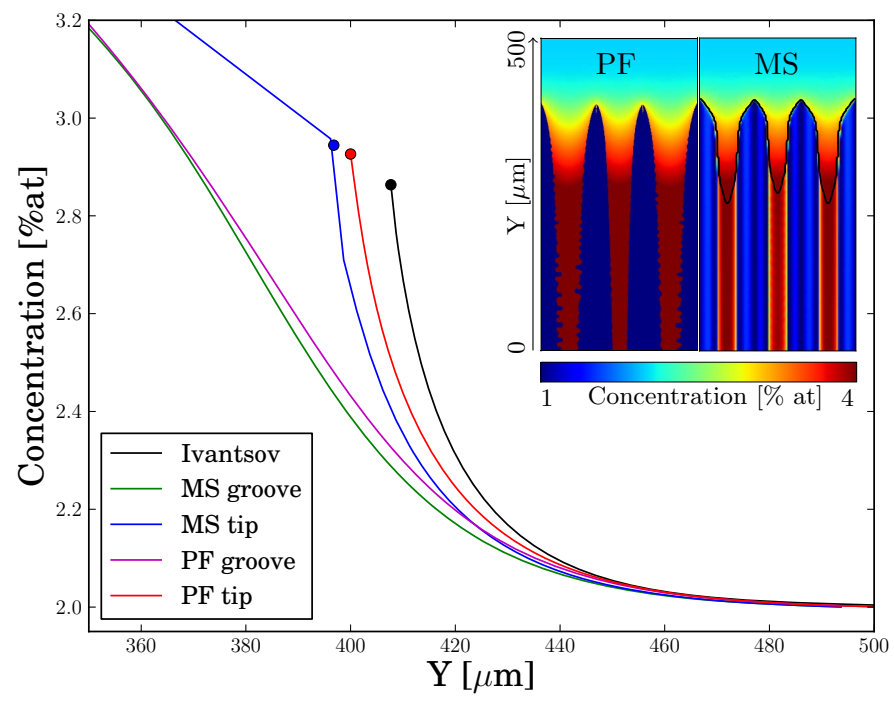

Fig. 2. Liquid concentration profiles along the axis of a primary arm and along a groove between two arms. Comparison of a phase-field and a mesoscopic simulation at the steady state $(t=50 \mathrm{~s})$. The Ivantsov solution for a free tip growing at the pulling speed is also shown for comparison. The inset shows the full concentration fields from the phase-field (PF) and mesoscopic (MS) simulations.

concentration $C_{0}$ without accounting for segregation effects: $\bar{g}_{\mathrm{s}}^{\text {Scheil }}(y)=1-\left[\left(T(y)-T_{\mathrm{f}}\right) /\left(m_{\mathrm{L}} C_{0}\right)\right]^{1 /(k-1)}$.

Both numerical solutions differ from the closed-system Scheil solidification path for two reasons. The strongest difference appears in the region just behind the primary tips. It occurs because the liquid is far from the equilibrium concentration in the envelope, $C_{1}^{*}(y)=\left(T(y)-T_{\mathrm{f}}\right) / m_{\mathrm{L}}$, that is assumed by the Scheil equation. This can be seen in Fig. 1,

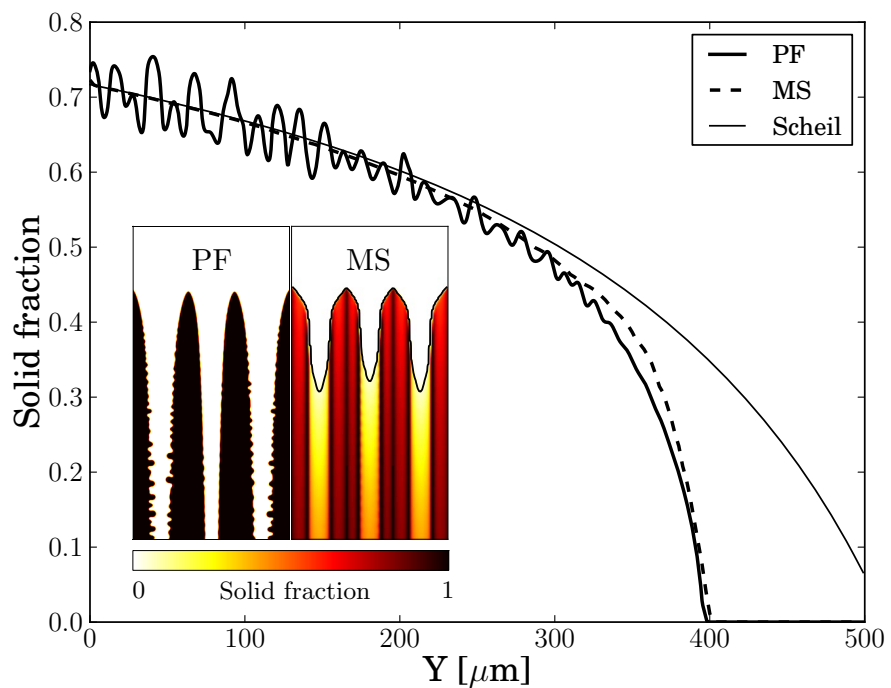

Fig. 3. Average solid-fraction profiles along the height of the mushy zone for phase field (PF) and mesoscopic (MS) simulations, and for the Scheil model. The inset shows the full solid fraction fields from both simulations.

where we clearly see the diffusion field in the liquid around the primary branches, and in Fig. 2, where we can see that the concentration in the groove is much lower than the equilibrium concentration. A smaller difference of the solid fraction can be seen to persist up to $300 \mu \mathrm{m}$ behind the tips. The reason for this difference is segregation of solute that transports solute by diffusion through the mushy zone towards the tips. This segregation at the mesoscopic scale results in an increased average solute concentration $\left(\bar{C}>C_{0}\right)$ behind the tips and thus delays the solidification. Both phenomena 
(a) Rebranching and elimination of a branch.

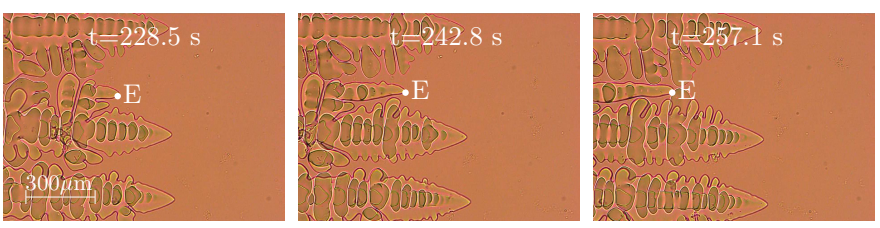

(b) Generation of primary branches by tertiary rebranching in a grain (P) and at a divergent grain boundary (R).
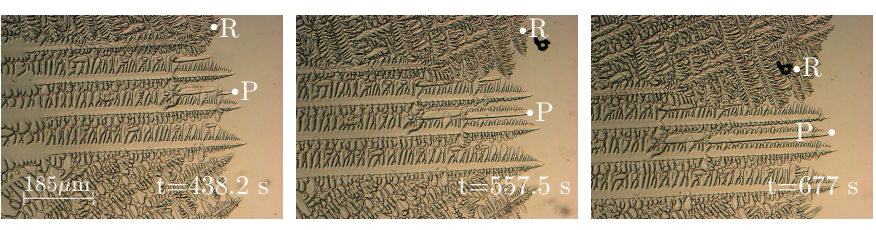

(c) Elimination of a branch at a converging grain boundary.
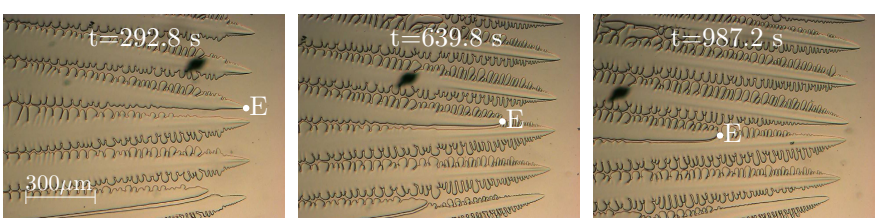

Fig. 4. Mechanisms of primary spacing adjustment. Micrographs of directional solidification of an NPG-TRIS (neopentyl glycoltris(hydroxymethyl)aminomethane) alloy, courtesy of Victor Witusiewicz (Access e.V.)

are accurately represented by the mesoscopic model.

\section{Adjustments of primary spacing and their dynamics}

At given conditions of directional solidification (in the Bridgman configuration defined by the temperature gradient and by the pulling speed) a finite range of stable primary dendrite arm spacings exists in columnar growth. The ultimate primary spacing at which the columnar pattern stabilizes does not depend exclusively on the operating conditions. It also depends on the initial conditions and on the history of the spacing selection. This has been shown by experiments [7, 15, 23, 24, 50], theoretical analyses [25, 48, 49], and models [31, 42, 47]. If the spacing is not within the stable range it can adjust in several different ways. The adjustment mechanisms are illustrated in Fig. 4. If the spacing is too small, a primary branch is overgrown by the neighboring branches and is thus eliminated (Fig. 4a). A too large spacing leads to tertiary rebranching that generates new primary branches (Fig. 4b). Locally nonuniform spacings can also adjust by a lateral drift of the branches.

An indication that the mesoscopic model can reproduce certain spacing adjustments was already given by the simulation shown in Fig. 1, where a tertiary rebranching occured. We now want to examine more systematically with what fidelity the different types of primary spacing adjustments and their dynamics can be predicted. The answer is far from being obvious because of the simplifying assumptions incorporated in the mesoscopic model. While the phenomena underlying spacing adjustment are essentially transient, the meso- scopic model uses a tip growth dynamics law that assumes steady-state diffusion at the scale of the stagnant-film and a constant tip selection parameter.

We performed phase-field and mesoscopic simulations with six different initial nucleus spacings, ranging from well below to well above the range of stable spacings. We also performed mesoscopic simulations initialized by a plane envelope, which can in this context be interpreted as an envelope of very closely spaced branches. Because of the lateral boundaries, the transient spacing adjustment process and the steady-state spacing are affected by the size of the system. The simulations were therefore done for three different domain widths: $250 \mu \mathrm{m}, 500 \mu \mathrm{m}$, and $1000 \mu \mathrm{m}$. This corresponds to around 3, 6, and 12 primary branches across the domain, respectively. The simulations in Section 2 have shown that the final stable spacing can be expected to be of the order of $100 \mu \mathrm{m}$.

The dynamics of the spacing adjustments predicted by both models is shown in detail for two representative cases. In the first case, the initial spacing of $1000 \mu \mathrm{m}$ is above the stability range and much larger than the final stable spacing. Tertiary rebranching needs to occur. In the second case, the initial spacing of $50 \mu \mathrm{m}$ is below the stability range and smaller than the final spacing. Certain branches thus need to be eliminated. In both cases the domain width is $1000 \mu \mathrm{m}$ and the final stable spacing is of the order of $100 \mu \mathrm{m}$.

Finally, the influence of the stagnant-film thickness on the predictions of the primary spacing and on the spacing adjustment dynamics was investigated. Where not stated otherwise, the stagnant-film thickness for the mesoscopic model was $\delta_{c}=1.18 l_{\text {diff }}$, where $l_{\text {diff }}=D_{1} / V_{\text {pull }}=2.2 \cdot 10^{-5} \mathrm{~m}$ is the diffusion length at the tips.

\subsection{Spacing adjustment by tertiary branching $\left(\lambda_{0}>\lambda_{1}\right)$}

The simulation of a configuration with an initial spacing larger than the final primary spacing is shown in Fig. 5 (left). The phase-field (PF) and mesoscopic (MS) simulations are shown side by side. The growth was initiated by two nuclei placed in the bottom corners of the domain. The initial spacing is thus $\lambda_{0}=1000 \mu \mathrm{m}$. In the phase-field simulation we first see a rapid growth of the lateral dendritic arms along the bottom domain boundary $(t=2 \mathrm{~s})$. Following a destabilization, numerous secondary arms evolve and grow along the temperature gradient. Their spacing is initially very small $(t=4 \mathrm{~s})$. Between 4 and $8 \mathrm{~s}$, a competition between these new branches takes place, eliminating most of them. 13 branches survive and the system then evolves to a steady state with a rather uniform spacing, shown in the last frame $(t=50 \mathrm{~s})$.

In the mesoscopic simulation the appearance of the vertical secondary branches is mimicked by a vertical spread of the envelope that corresponds to the lateral dendrite arms $(t=4 \mathrm{~s})$. The vertically expanding envelope is smooth at this stage. Later on it is gradually destabilized by instabilities that propagate both from the groove in the middle, where the horizontal arms met, and from the domain boundaries $(t=8-10 \mathrm{~s})$. The instabilities evolve into a steady pattern 
$\lambda_{0}=1000 \mu \mathrm{m}$
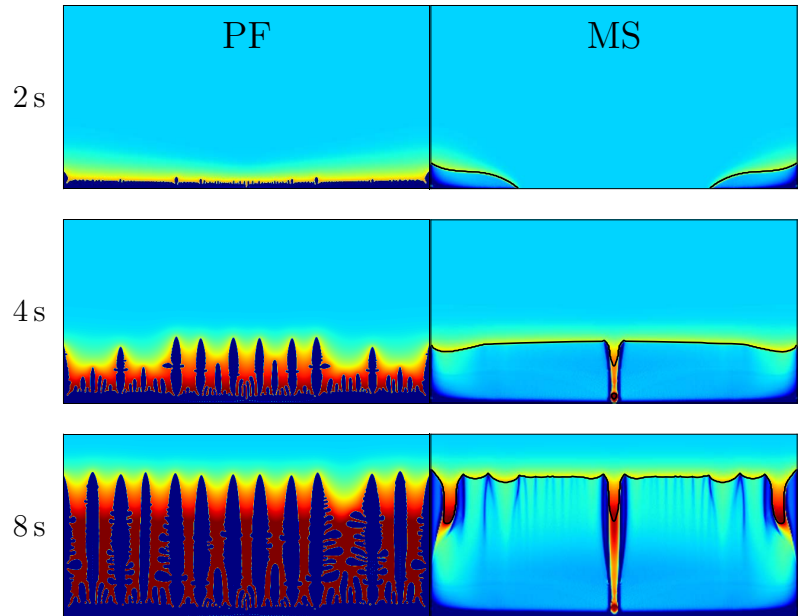

$10 \mathrm{~s}$

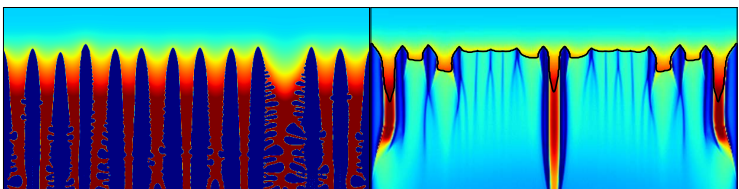

$50 \mathrm{~s}$

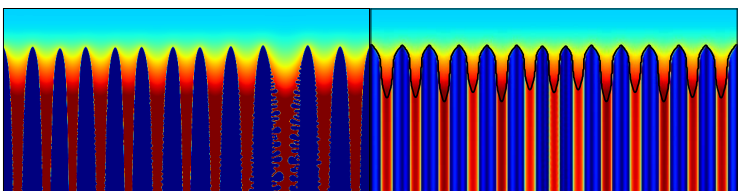

$\lambda_{0}=50 \mu \mathrm{m}$
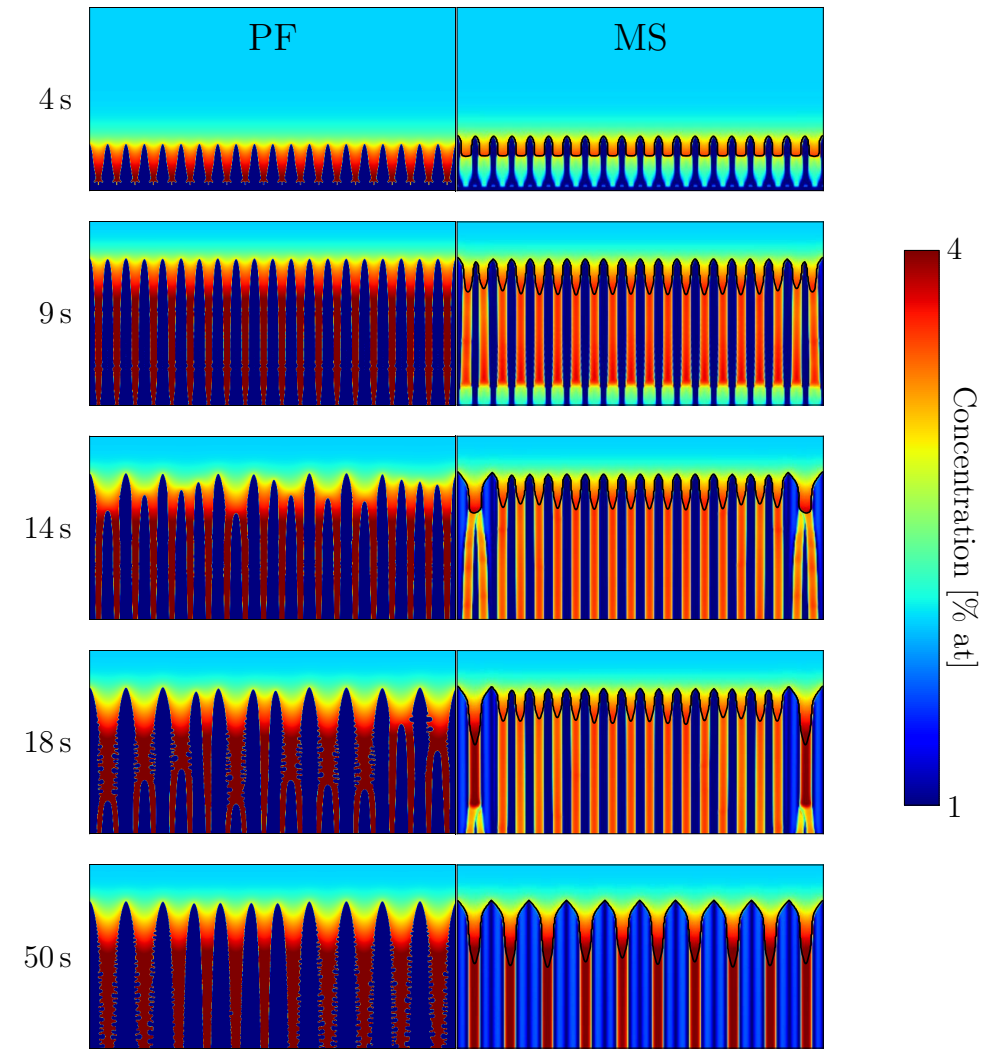

Fig. 5. The evolution of the columnar grains from an initial spacing of $\lambda_{0}=1000 \mu \mathrm{m}$ (left column) and $\lambda_{0}=50 \mu \mathrm{m}$ (right column). The domain size is $500 \times 1000 \mu \mathrm{m}$. The concentration field is shown for phase field (PF) and the average concentration field and the envelope (black line) are shown for the mesoscopic model (MS).

of well pronounced primary branches $(t=50 \mathrm{~s})$. At steady state, the arrangement of primary arms is slightly nonuniform and the primary dendrite spacing is stable with a mean value of $\lambda_{1}^{\mathrm{MS}}=77 \mu \mathrm{m}$. This is very close to the mean primary dendrite spacing predicted by phase field: $\lambda_{1}^{\mathrm{PF}}=83 \mu \mathrm{m}$. The time to obtain a constant number of primary arms is around $t=8 \mathrm{~s}$ for the phase-field and $t=14 \mathrm{~s}$ for the mesoscopic model.

The horizontal growth of the initial lateral branches in the mesoscopic simulation is significantly slower than in the phase-field simulation ( $t=2 \mathrm{~s}$ ). These differences are mainly due to the fact that the mesoscopic model assumes a constant tip selection parameter, $\sigma^{*}$, whereas in the phase-field model the initial instabilities freely evolve without assuming steady state dynamics. We calculated the selection parameter from the tip radius and the velocity of the lateral arms, measured in the phase-field simulations. We could see that it varies from $\sigma^{*}=0.30$ at $0.5 \mathrm{~s}$ to $\sigma^{*}=0.07$ at $1.5 \mathrm{~s}$. Although the decay is quick, $\sigma^{*}$ is an order of magnitude larger at the growth onset than in the steady state. The resulting velocity increase is enormous (see Eq. (2)). It is only weakly counteracted by two additional effects that are not accounted for in the mesoscopic model: the kinetic and capillary undercooling are much larger than in the steady state, both are of the order of $0.3 \mathrm{~K}$. The effect of the difference between the seed size in the two models [33] is only minor here. Regardless of the differences in the initial lateral growth, the vertical growth of the dendrite front agrees very well with that predicted by phase field. The advancement of the columnar front - indicated by the position of the most advanced primary tip - during the initial transient is plotted in Fig. 6 (plot MS $\left(\delta_{c}=1.18 l_{\text {diff }}\right)$ ). The plot shows that the mesoscopic model accurately predicts the global dynamics of the solidification zone.

Despite a good agreement of the steady-state growth patterns given by both models, the sequence of events adjusting a spacing above the stability limit to a stable spacing is clearly different. In the mesoscopic simulation the adjustment process depends on the stagnant-film thickness $\delta_{c}$, a model parameter. The phase-field model first shows the emergence of numerous new branches with a fine spacing. In a second phase a very fast elimination of branches occurs, leading to an early stabilization of the average primary spacing. The mesoscopic model does not develop any apparent secondary branches from the lateral arms. Instead, a smooth envelope spreads out vertically. This is however interpreted as an envelope of vertically growing secondary arms. Only in a second phase, this smooth envelope is destabilized and finely spaced perturbations appear, implying a branched structure. The elimination of some of these perturbations and the devel- 


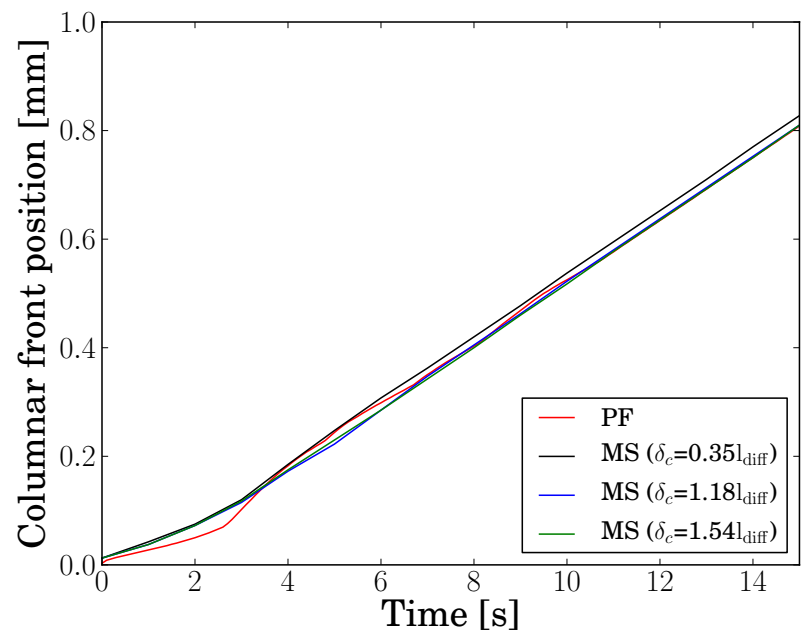

Fig. 6. Evolution of the columnar front position in the phase-field (PF) and mesoscopic (MS) simulations for $\lambda_{0}=1000 \mu \mathrm{m}$. The results for $\lambda_{0}=50 \mu \mathrm{m}$ are similar and are not shown to avoid overlap. The results of the mesoscopic model are from simulations with three different stagnant-film thicknesses, $\delta_{c}$.

opment of a stable primary branch pattern then develops in a cascade, departing from the boundaries of the domain and from the extremities of the lateral branches in the middle of the domain. Fig. 7a compares the development of the average spacing with time (see plot MS $\left(\delta_{c}=1.18 l_{\text {diff }}\right.$ ) for the mesoscopic model). We can see that the adjustment of the average spacing shows a similar dynamics, but takes about twice as much time as predicted by the phase-field model. As indicated by additional curves shown in the graph, this evolution also depends on the stagnant-film thickness, which will be discussed in detail later in this section.

\subsection{Spacing adjustment by branch elimination $\left(\lambda_{0}<\lambda_{1}\right)$}

The case with a configuration of an initial spacing smaller than the final primary arm spacing is given on the right side of Fig. 5. The growth was initiated by 20 nuclei placed along the bottom boundary of the domain with a spacing of $\lambda_{0}=50 \mu \mathrm{m}$. All nuclei give rise to vertically growing primary branches. The growth of lateral branches is hindered by the small initial spacing. The advancement of the columnar front with time is similar to the case of $\lambda_{0}>\lambda_{1}$ (Fig. 6) and is again virtually identical for both models. The predictions of both models concerning the transient stage are different. The phase-field model (PF) predicts a fast spacing adjustment that proceeds by a sudden elimination of vertical branches, which resembles a period doubling instability [24]. The process starts at $t=12 \mathrm{~s}$ and is finished by $t=18 \mathrm{~s}$. The mesoscopic model (MS) gives an entirely different adjustment process. The spacing adjustment proceeds symmetrically from the left and right edges of the domain by elimination of vertical branches in a cascade. The final spacing is stabilized only at $t=37 \mathrm{~s}$. The adjustment process thus takes three times longer than in the phase-field simulation. The evolution of the average spacing is shown in Fig. 7b (see plot MS $\left(\delta_{c}=1.18 l_{\text {diff }}\right)$ for the mesoscopic model). Nev- (a) $\lambda_{0}>\lambda_{1}$

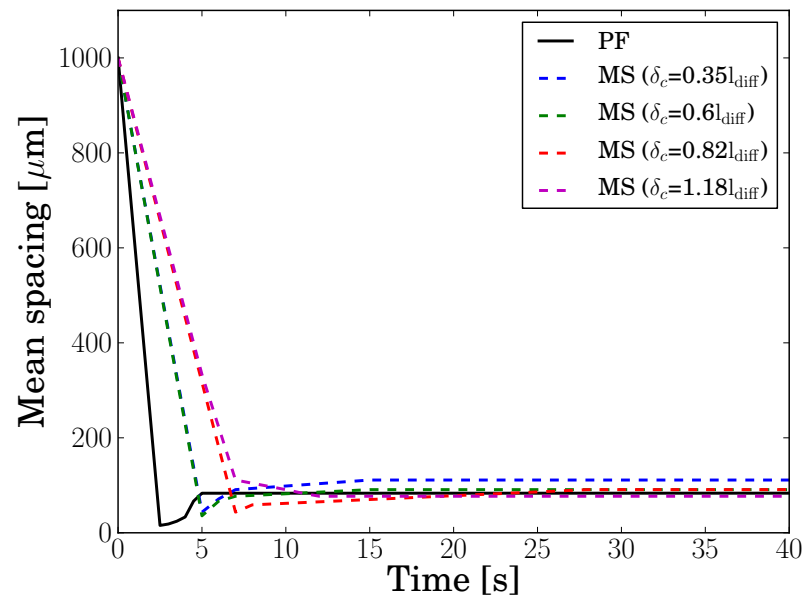

(b) $\lambda_{0}<\lambda_{1}$

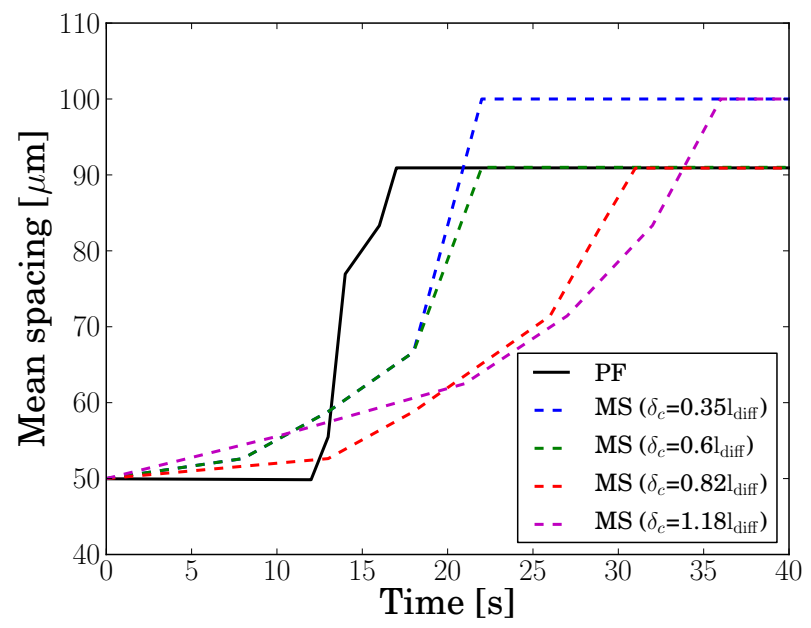

Fig. 7. Evolution of the average primary arm spacing in the phase-field and mesoscopic simulations for two initial spacings, $\lambda_{0}$. (a) $\lambda_{0}=1000 \mu \mathrm{m}$, (b) $\lambda_{0}=50 \mu \mathrm{m}$. The results of the mesoscopic model are from simulations with three different stagnant-film thicknesses, $\delta_{c}$.

ertheless, the predicted final mean spacings are very close: $\lambda_{1}^{\mathrm{MS}}=100 \mu \mathrm{m}$ and $\lambda_{1}^{\mathrm{PF}}=91 \mu \mathrm{m}$.

In Fig. 8, the final spacing, $\lambda_{1}$, is shown for all simulated configurations. It is plotted as a function of the initial spacing, $\lambda_{0}$, ranging from 0 (initially planar envelope) to $1000 \mu \mathrm{m}$, for three different lateral confinements. Since the final spacing depends on the history of the system, both parameters have an influence. The predicted spacings are compared to classical analytical geometrical models, which give a unique average primary spacing for steady growth as a function of the temperature gradient, the pulling velocity and the solute concentration. They can be summarized by $\lambda_{1}=a \sqrt{\ell_{\mathrm{sl}} R_{\text {tip }}}$, where $\ell_{\mathrm{sl}}$ is the total depth of the mushy zone, $R_{\text {tip }}$ is the tip radius and where the constant $a$ is alloyspecific [35]. Hunt's model [16] is based on the minimum undercooling. Kurz and Fisher [19] developed a model that assumes that the shape of the dendrite can be approximated as an ellipsoid of revolution and used the marginal stability criterion for an isolated dendrite. Trivedi [45] modified Hunt's 


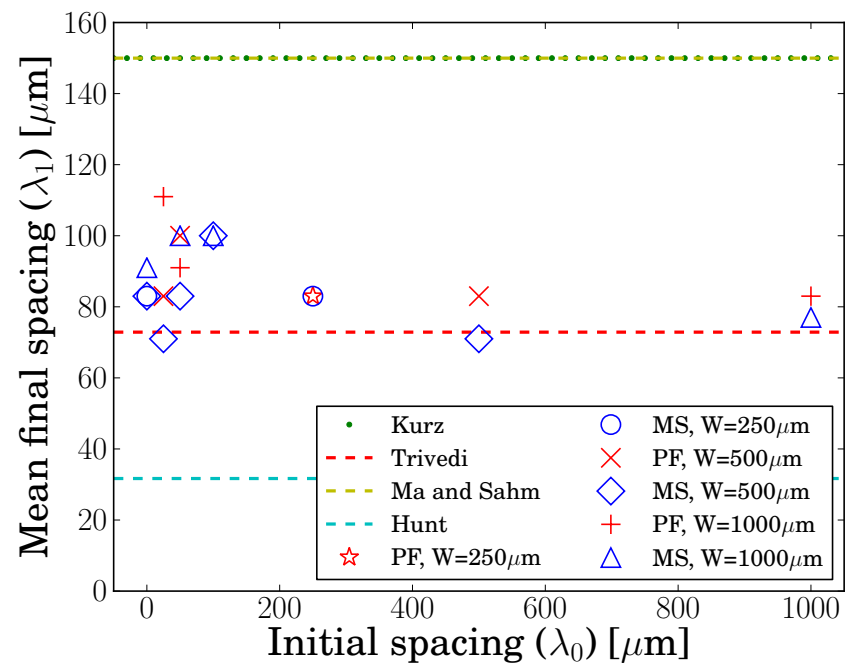

Fig. 8. Dependence of the final primary arm spacing on the spacing of the initial nuclei, $\lambda_{0}$, and on the width of the confinement $W$. Mesoscopic simulations with an initially planar front are characterized by an initial spacing of $\lambda_{0}=0$. All MS results are from simulations with $\delta_{c}=1.18 l_{\text {diff }}$.

model using the marginal stability criterion. The model of Ma and Sahm [26] takes in account the effect of secondary arms. These models predict primary spacings between $32 \mu \mathrm{m}$ and $150 \mu \mathrm{m}$. This is a very wide range and all the predictions of the mesoscopic and the phase-field model fall within this range.

\subsection{The influence of the stagnant-film thickness}

The results of the mesoscopic model depend to a certain degree on the principal model parameter, the stagnant-film thickness $\delta_{c}$ [30]. In order to analyze the sensitivity in simulations of columnar growth, we performed computations for six different values of $\delta_{c}$. All were chosen to be close to $\delta_{c}=l_{\text {diff, }}$, which has been shown to be a satisfactory calibration in situations of free equiaxed growth [30].

Fig. 9 shows spatiotemporal plots of the simulations for two initial spacings: $\lambda_{0}=1000 \mu \mathrm{m}$ and $\lambda_{0}=50 \mu \mathrm{m}$. The most striking is the influence of $\delta_{c}$ on the amplitude of the wavy envelope pattern and on the oscillatory pattern in the solid-fraction field within the envelope. Both weaken as $\delta_{c}$ is increased. For large $\delta_{c}$ the oscillations that represent the primary branch pattern become very weak $\left(\delta_{c}=1.54 l_{\text {diff }}\right)$. For sufficiently high values the oscillations almost entirely disappear $\left(\delta_{c}=1.77 l_{\text {diff }}\right.$ ) and the envelope tends towards a smooth planar front. Only very weak traces in the solidfraction field still reveal a pattern. Generally, oscillations of the solid-fraction are stronger when the cells of the wavy envelope pattern are deeper and when the tip of the envelope cell is sharper. It is not difficult to understand that the solute diffusion flux from a convex-shaped section of the envelope is larger than from a plane section. We can apply a solute balance at the envelope:

$$
(1-k) C_{\mathrm{l}, \text { env }}^{*} g_{\mathrm{s}, \mathrm{env}} v_{n}=D_{\mathrm{l}}\left(\left.\frac{\partial C_{\mathrm{l}}}{\partial n}\right|_{1}-g_{\mathrm{s}, \mathrm{env}} \frac{\nabla T}{m_{\mathrm{L}}} \cdot \vec{n}\right),
$$

Table 2

Influence of the stagnant-film thickness, $\delta_{c} / l_{\text {diff }}$, on the final primary arm spacing. All results are for a domain width of $W=1000 \mu \mathrm{m}$.

\begin{tabular}{lrrr}
\hline & $\delta_{c} / l_{\text {diff }}$ & $\lambda_{0}=1000 \mu \mathrm{m}$ & $\lambda_{0}=50 \mu \mathrm{m}$ \\
\hline Phase field & - & $\mathbf{8 3}$ & $\mathbf{9 1}$ \\
\hline Mesoscopic & 0.35 & $\mathbf{1 1 1}$ & $\mathbf{1 0 0}$ \\
& 0.59 & $\mathbf{1 0 0}$ & $\mathbf{9 1}$ \\
& 0.82 & $\mathbf{9 1}$ & $\mathbf{9 1}$ \\
& 1.18 & 77 & $\mathbf{1 0 0}$ \\
& 1.54 & $\mathbf{8 3}$ & $\mathbf{7 1}$ \\
& 1.77 & $\mathbf{3 4}$ & $\mathbf{3 1}$ \\
\hline
\end{tabular}

where $C_{1, \text { env }}^{*}$ and $g_{\text {s,env }}$ are the equilibrium liquid concentration and the solid fraction at the envelope, respectively. The terms on the right hand side of the equation are the diffusion fluxes at the outer (liquid) side and at the inner side of the envelope, respectively. We can see that for a given orientation of the envelope $(\vec{n})$ and a given position in the temperature gradient $\left(C_{1, \text { env }}^{*}\right)$, the solid fraction that forms at the envelope only depends on the diffusion flux of solute into the liquid. Because more solute can be rejected into the liquid when a protuberance is present, more solid forms directly at the envelope front.

Furthermore, we can easily notice that the spacing adjustment process becomes slower with increasing $\delta_{c}$. The evolution of the average spacing for the four smallest $\delta_{c}$ is shown in Fig. 7 and the final spacings are summarized in Table 2. In the case of spacing adjustment by rebranching (Fig. $7 \mathrm{a}$, $\lambda_{0}=1000 \mu \mathrm{m}$ ), we can see that an increase of $\delta_{c}$ decreases the final spacing, $\lambda_{1}$. From $\lambda_{1}=111 \mu \mathrm{m}$ for $\delta_{c}=0.35 l_{\text {diff }}$ it decreases to $\lambda_{1}=77 \mu \mathrm{m}$ for $\delta_{c}=1.18 l_{\text {diff. }}$. For the case of spacing adjustment by branch elimination (Fig. 7b, $\lambda_{0}=50 \mu \mathrm{m}$ ) the final spacing remains almost constant, varying between 91 and $100 \mu \mathrm{m}$. In both configurations (with elimination and with rebranching), the protuberances of the envelope indicating primary arms almost vanish for the two largest $\delta_{c}, 1.54 l_{\text {diff }}$ and $1.77 l_{\text {diff. The final average spacing }}$ also decreases sharply as $\delta_{c}$ approaches $2 l_{\text {diff }}$.

The formation of the envelope pattern is caused by a diffusion-driven front instability and a larger $\delta_{c}$ seems to be a stabilizing factor. Overall, a smaller $\delta_{c}$ appears to accelerate the spacing adjustment process. Figs. 7a and 9 show that with the initial spacing of $\lambda_{0}=1000 \mu \mathrm{m}$, the destabilization and rebranching of the envelope occur earlier and that the branches are more finely spaced as $\delta_{c}$ is decreased. In both cases, the adjustment from a too fine to a coarser spacing is also faster when $\delta_{c}$ is smaller (Figs. 7a, 7b and 9). Fig. 6 shows that the advancement of the columnar front with time does not depend on the stagnant-film thickness. In summary, stagnant-film thicknesses of the order of $0.5 l_{\text {diff }} \lesssim \delta_{c} \lesssim l_{\text {diff }}$ give a good estimation of the primary spacing and of the spacing adjustment dynamics.

Nevertheless, the time scales for adjustment of the primary spacing are significantly longer in the mesoscopic simulations 


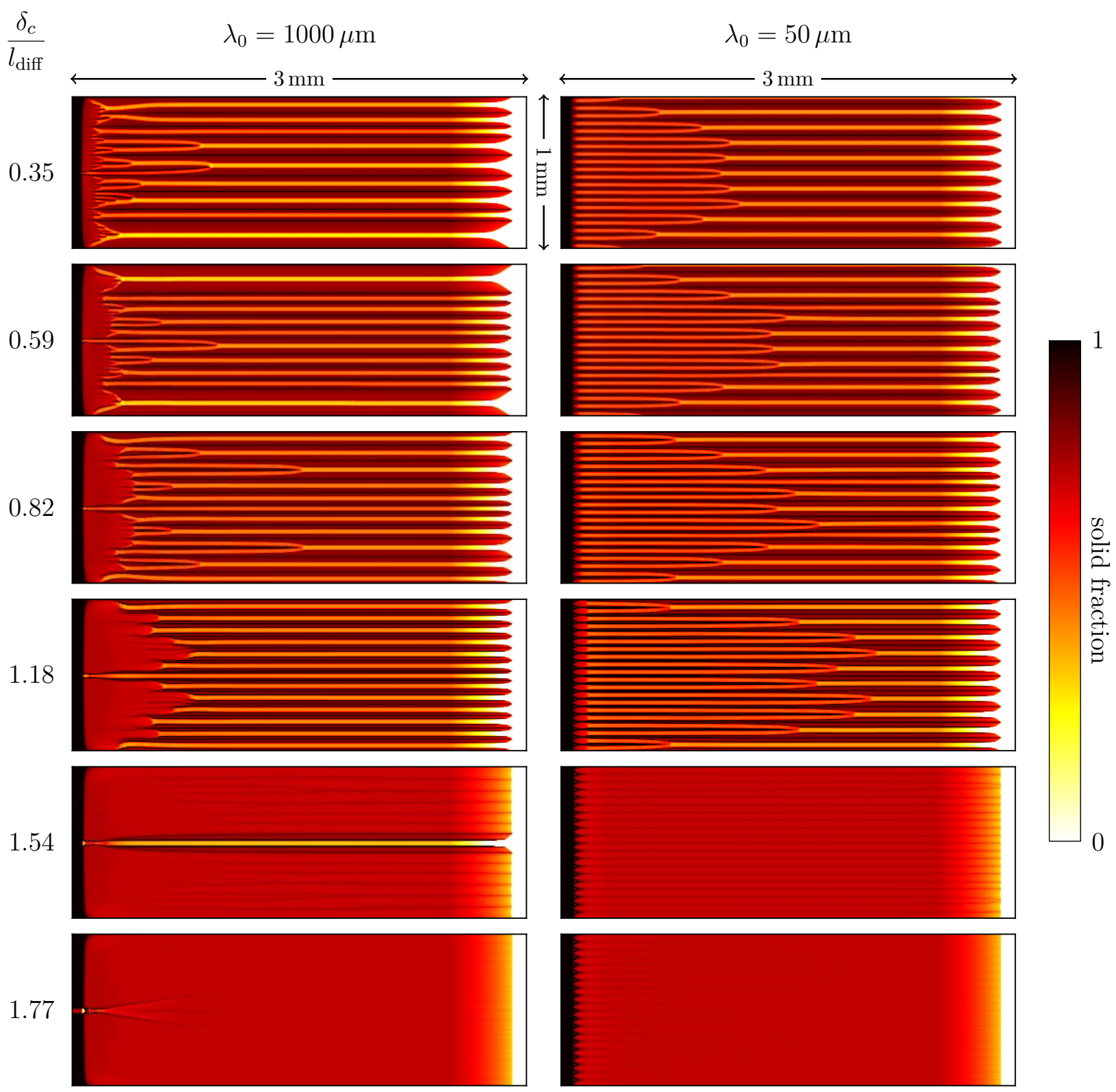

Fig. 9. Spatiotemporal plots of mesoscopic simulations with different stagnant-film thicknesses, $\delta_{c}$. The plots show the solid fraction and were reconstructed from the moving-frame simulations up to $t=50 \mathrm{~s}$.

and the sequences of individual adjustment events are clearly different from the phase-field predictions. The most probable reason for the disparities is the approximation of the tip growth dynamics by a steady-state law. The accuracy of such an approximation has been quantified for transients in free equiaxed growth [33]. It remains to be clarified within which limits of solidification parameters (temperature gradient, cooling rate, initial spacing) this law can provide quantitative predictions of the transients in columnar growth. This aspect requires further investigation but is not within the scope of the current article.

\section{Growth competition of misoriented grains}

The texture of polycrystalline columnar structures is an important macrostructural parameter. Since the representation of interactions in large ensembles of growing grains is the main purpose of the mesoscopic solidification model, an investigation into its predictive capabilities in polycrystalline growth is pivotal. A prototypal configuration of such growth is shown in Fig. 10. Three dendritic grains are growing directionally, pulled at a constant rate in a constant temperature gradient. The grain whose crystal orientation is inclined by an angle $\alpha_{0}$ with respect to the temperature gradient (misoriented grain) grows between two grains that are aligned with the temperature gradient (well-oriented grains). Two grain boundaries (GBs) form, referred to as diverging when 
$1 \mathrm{~mm}$

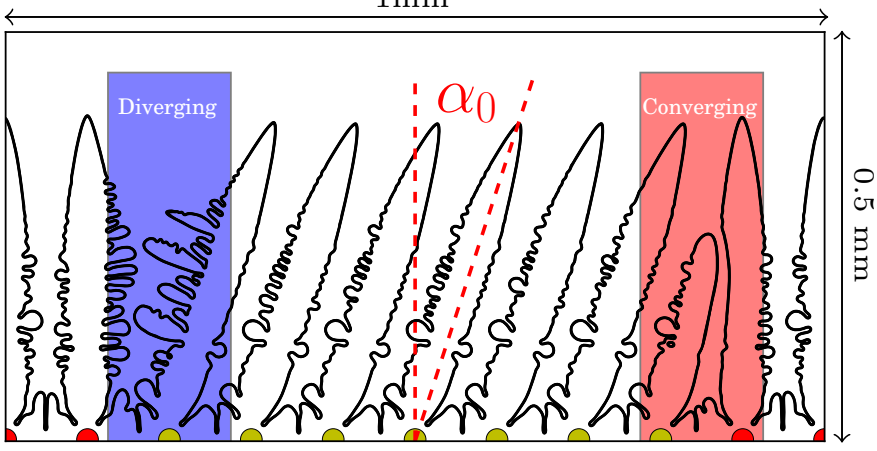

Fig. 10. Schematics of the calculation domain and of the initial conditions. The semicircles represent the initial seeds and their crystal orientations: yellow and red for the misoriented and the well-oriented grains, respectively.

the primary branches grow away from each other and as converging when they grow towards each other. The interactions of the dendrite branches adjacent to the grain boundaries at the scale of the primary spacing govern the migration of the grain boundaries at a much larger mesoscopic scale. Depending on the difference of the angles of the grain-boundary trajectories, both grains can coexist or a grain can be eliminated within a certain characteristic distance.

The evolution of a diverging GB (blue area in Fig. 10) is governed by tertiary rebranching that occurs once the spacing between the two adjacent grains becomes large enough to develop a significantly supersaturated gap. The GB angle $\theta_{\mathrm{D}}$ depends on the side on which tertiary rebranching occurs. If only the misoriented grain rebranches (Fig. 4b, tip "R"), the GB remains aligned with the temperature gradient. If the well-oriented grain rebranches, this blocks the rebranching from the misoriented grain and the diverging GB deviates from the direction of the temperature gradient. The evolution of a converging GB (red area in Fig. 10) is governed by an overgrowth mechanism that eliminates a primary branch of either the misoriented or the well-oriented grain, once the tips grow too close. At a diverging GB the well-oriented grain is always favored at a mesoscopic scale, at a converging GB either grain can win. The GB angle $\theta_{C}$ is usually much smaller than that of a diverging GB, $\theta_{\mathrm{D}}$ and is often close to zero. These grain competition patterns have been observed in many experiments [13, 46, 53].

Tourret and Karma [44] recently studied grain competition of columnar dendritic grains by 2D phase-field simulations. They have shown that the selection of the trajectory of the diverging GB is stochastic. The reason for this is that the branching dynamics from which it originates is chaotic and critically depends on the thermal noise around the dendrite tip. However, despite the stochasticity, statistically clear trends of the GB angle as a function of the control parameters (temperature gradient, pulling speed, grain inclination angle) were observed. In contrast to this, the adjustments of the intragrain primary spacing through tertiary branching are much less sensitive to the chaotic nature of the branching process. The same is observed for converging GBs.

Our objective is to investigate whether and how the meso-

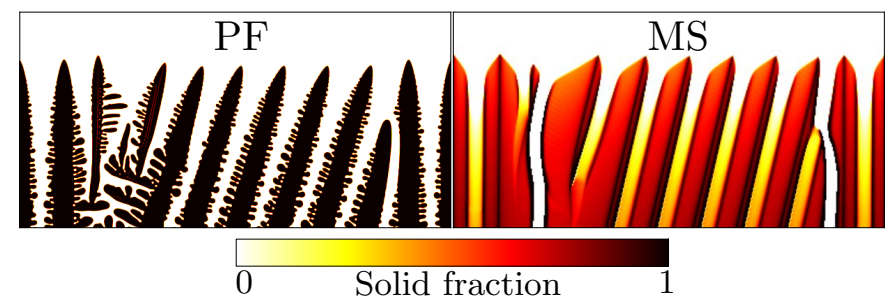

Fig. 11. Dendrite structure given by the phase-field (PF) and by the mesoscopic model (MS) for $\alpha_{0}=20^{\circ}$ at $t=20 \mathrm{~s}$.

scopic model, which uses a simplified representation of dendrites by envelopes and describes their dynamics by simplified tip kinetics laws, can reproduce the process of GB evolution. We compare mesoscopic and phase-field simulations of the prototypal configuration shown in Fig. 10. The phasefield model that we used as a reference does not include thermal noise and is thus deterministic. It should nevertheless give trends of GB selection that are the same as those obtained statistically by stochastic phase-field models. The results are illustrative for the GB selection process since we tested only one configuration, i.e., we did not vary the initial position of seeds or the width of the domain. We would expect that this could affect the morphological evolution. As shown in Fig. 10, the growth is initiated at the bottom of the domain from semicircular nuclei: two well-oriented nuclei on each side and seven misoriented nuclei in between. The spacing between the nuclei is $100 \mu \mathrm{m}$, which is within the stable spacing range for the growth of a single well-aligned grain (see preceding sections). The crystalline misorientation angle $\alpha_{0}$ was varied between $5^{\circ}$ and $45^{\circ}$. All simulations were done on a moving-frame domain of $1000 \times 500 \mu \mathrm{m}^{2}$. All other physical, model and numerical parameters are the same as in the preceding simulations. The influence of the stagnant-film thickness in the mesoscopic simulations is also investigated.

Fig. 11 shows an example of a phase-field and a mesoscopic simulation of grain interaction. It shows three different GB competition phenomena: the rebranching of a welloriented grain and the rebranching of a misoriented grain at a diverging GB, and the elimination of a misoriented primary branch at a converging GB. We can see that the mesoscopic model can reproduce all three phenomena.

The full evolution of the microstructure for misorientation angles from $5^{\circ}$ and $45^{\circ}$ is shown in Fig. 12 in the form of spatiotemporal diagrams. Mesoscopic and phase-field simulations are compared for $200 \mathrm{~s}$ of simulated time. The length of the grains is of the order of 100 primary spacings.

For the phase field results we can observe that in a majority of simulations the misoriented grain is eliminated by the tertiary branching from the well oriented grain at the diverging GB. Both grains generate tertiary branches, but the well oriented branches prevail since they grow at a lower undercooling, i.e., ahead of the misoriented branches. After the misoriented grain is eliminated, the average spacing of the primary branches of the well-oriented grain stabilizes at 
$\alpha_{0}$

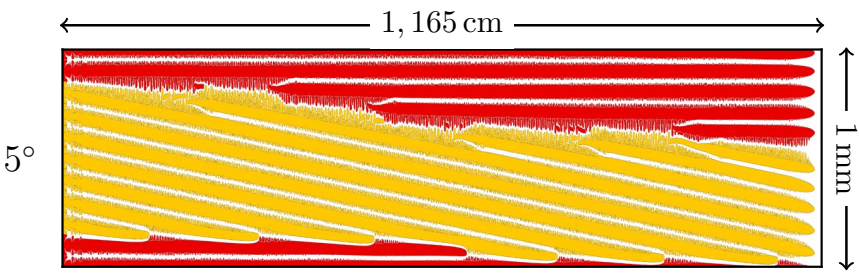

$10^{\circ}$

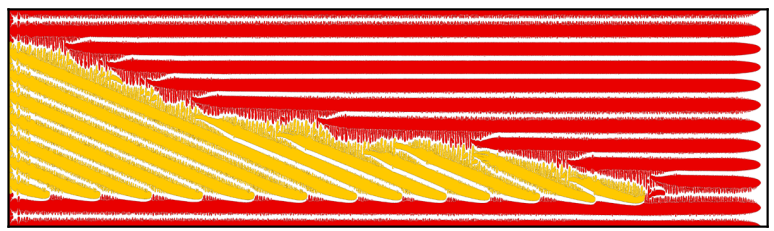

$15^{\circ}$

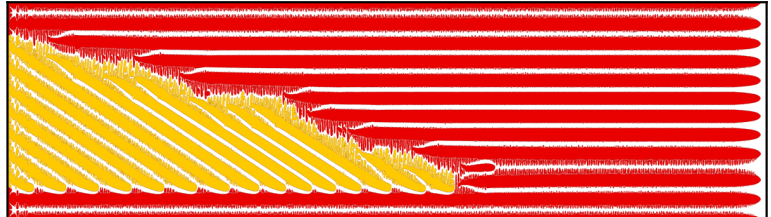

$20^{\circ}$

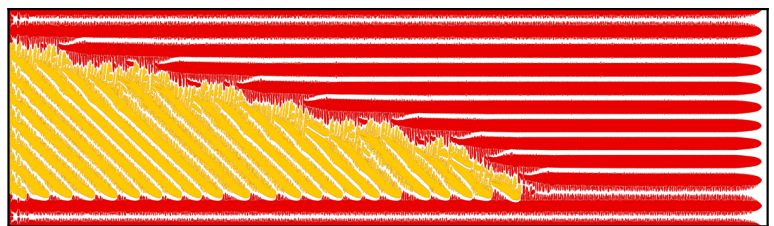

$25^{\circ}$

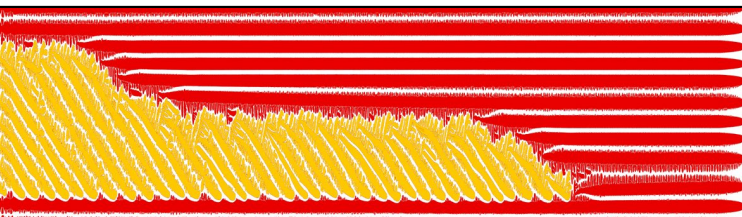

$30^{\circ}$

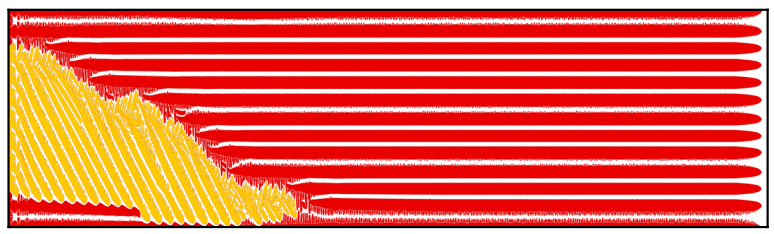

$35^{\circ}$

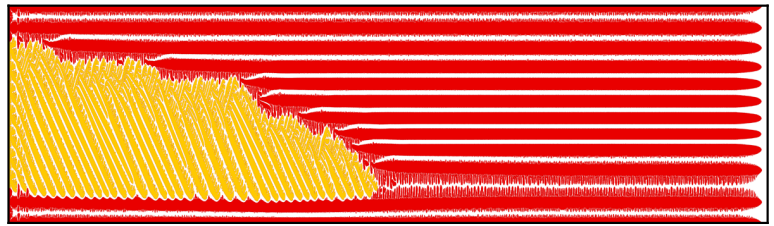

$40^{\circ}$

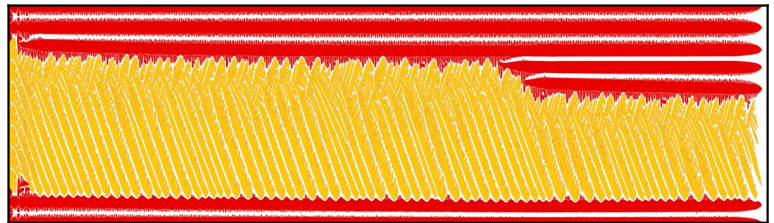

$45^{\circ}$

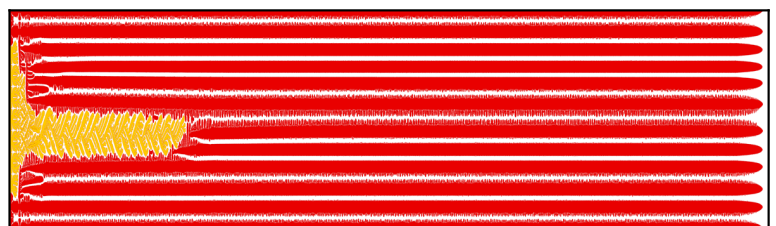

Mesoscale
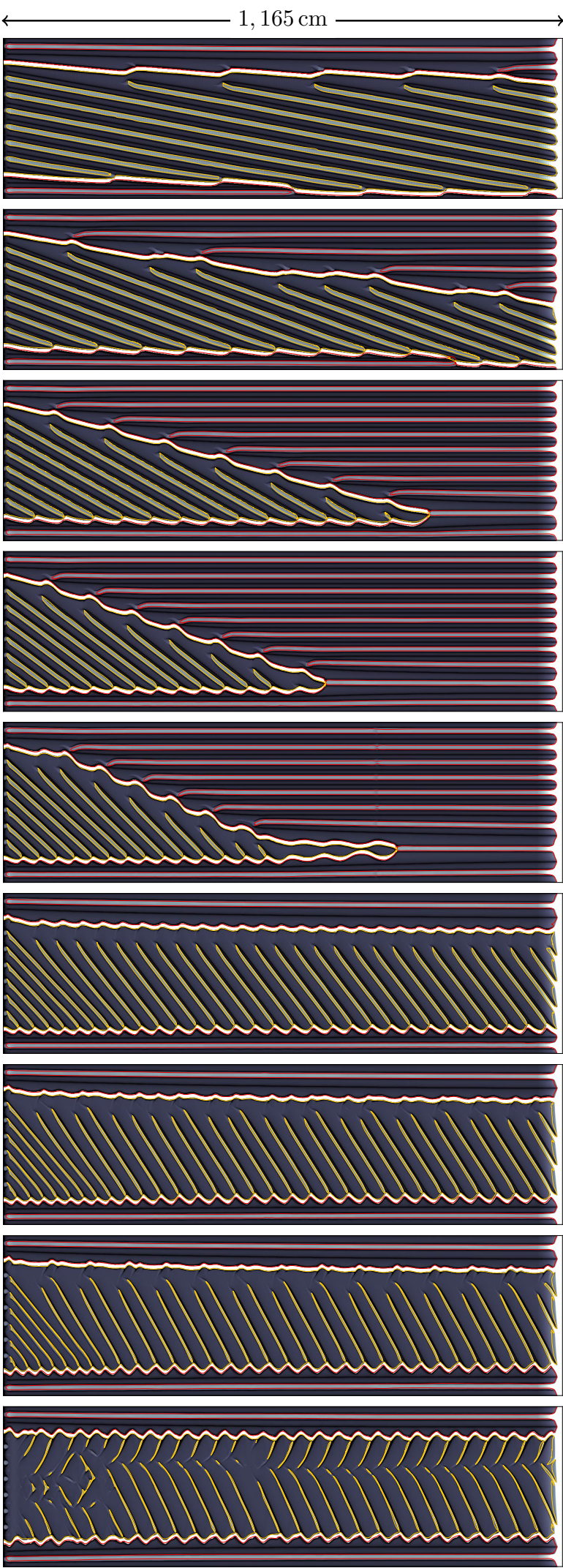

Fig. 12. Spatiotemporal plots of growth competition predicted by the phase-field (left) and the mesoscopic model (right). The well-oriented grains are shown in red and the grain misoriented by the angle $\alpha_{0}$ is shown in yellow (full for phase field, contour for mesoscale where solid fraction is superimposed). Note that the horizontal length is displayed compressed by a factor of 0.3 . 
$81 \mu \mathrm{m}$, identically in all cases. We can also remark that for misorientations below $20^{\circ}$, the distance needed to eliminate the misoriented grain becomes smaller with increasing misorientation. Such a trend was observed experimentally [53]. A fast elimination by branching at the diverging GB is also observed at $\alpha_{0}=30^{\circ}, 35^{\circ}$, and $45^{\circ}$. Nevertheless, the results at $\alpha_{0}=25^{\circ}$ and $40^{\circ}$ show that the misoriented grain can coexist with the well-oriented grain during a long time under some circumstances. Such behavior has also been observed experimentally [46]. The converging GB does not play a major role in the elimination of the misoriented grain. The converging GB angle is mostly zero or very close to zero. An elimination of the well-oriented grain is observed at $\alpha_{0}=5^{\circ}$ and $30^{\circ}$. Such anomalous overgrowth of the well-oriented by the misoriented grain has been observed in experiments [53] and in other phase-field simulations [22, 29, 39-41, 44, 51] at low misorientations, when the difference of the undercooling of the converging tips is low. In this case the undercooling of the well-oriented tip can drop below that of the misoriented tip if the local spacing of the well-oriented primary arms is sufficiently decreased by the pushing of the misoriented grain. At $\alpha_{0}=45^{\circ}$ both GBs are equivalent. Rebranching of the well oriented grain occurs at both GBs and the misoriented grain is eliminated very quickly. In the early stage of growth no obvious orientational order can be observed in the misoriented grain. The microstructure of the misoriented grain at $\alpha_{0}=45^{\circ}$ is not dendritic anymore, a transition to a degenerate growth is observed.

In the mesoscopic results, we can observe an elimination of the misoriented grain by tertiary branching from the well oriented grain for misorientations $\alpha_{0} \leq 25^{\circ}$. As in the phasefield simulations, both grains create new branches, but the well-oriented branches have an advantage in the competition. The average primary spacing of the well-oriented grain stabilizes between $100 \mu \mathrm{m}\left(\alpha_{0}=15^{\circ}\right.$ and $\left.20^{\circ}\right)$ and $111 \mu \mathrm{m}$ $\left(\alpha_{0}=25^{\circ}\right)$. The phenomenon of anomalous overgrowth is also described, an elimination of the well-oriented grain is observed at $\alpha_{0}=5^{\circ}$ and $10^{\circ}$. The elimination mechanism is similar to what is observed in the phase-field simulations. At misorientations of $30^{\circ}$ and higher, no tertiary branching is observed from the well-oriented grain. Only the misoriented grain generates new branches at the diverging GB. These branches do however not block the tips of the well-oriented grain and hence both grains continue to coexist. We can notice a slight shrinking of the width of the misoriented grain, but it is not clear whether this will only result into an adaptation of the primary spacing of both grains or whether the spacing increase of the well-oriented grain will eventually become large enough to trigger branching events. The rate of elimination of the misoriented grain can be expressed as an average angle between the divergent and the convergent GB, $\left(\theta_{\mathrm{D}}-\theta_{\mathrm{C}}\right)$. This angle is plotted in Fig. 13 as a function of the crystalline misorientation, $\alpha_{0}$. The inset shows how the two GB angles, $\theta_{\mathrm{D}}$ and $\theta_{\mathrm{C}}$, were measured. For small and moderate misorientations $\left(\alpha_{0}<30^{\circ}\right)$ the elimination angles given by the phase-field and the mesoscopic simulations show the same trend and differ less than $2.5^{\circ}$. For large misorienta-

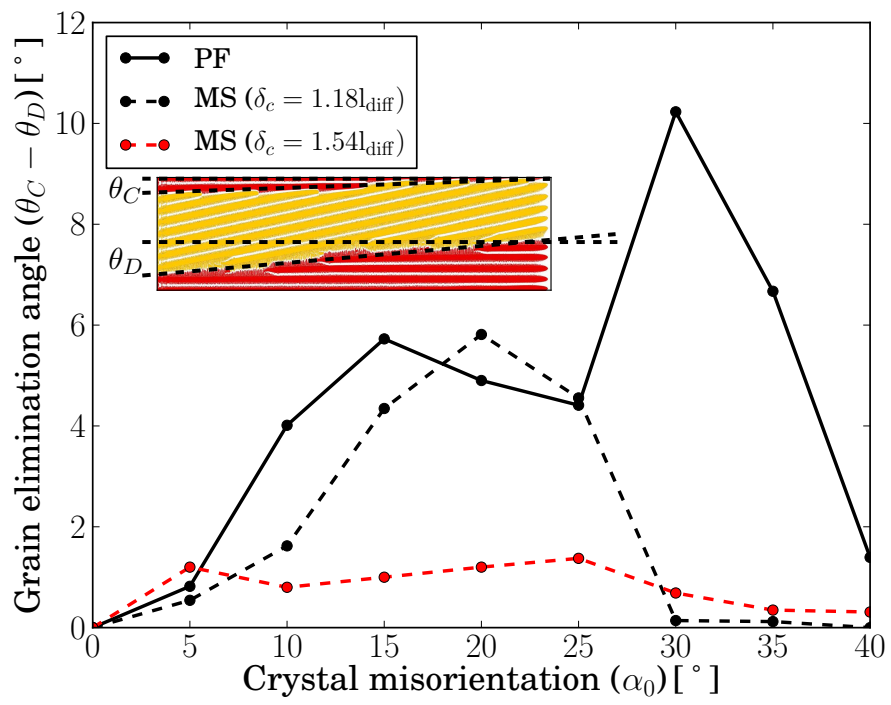

Fig. 13. Grain elimination angles as function of the crystal misorientation angle $\alpha_{0}$ of the misoriented grain. Comparison between mesoscopic (MS) simulations for two stagnant-film thicknesses and the phase-field (PF) simulations. Grain elimination angles for smaller stagnant-film thicknesses $\left(\delta_{c}<1.18 l_{\text {diff }}\right)$ in the MS model were zero.

tions, we observe substantial differences. The reason is that the mesoscopic model fails to predict the elimination of the misoriented grain. An exception is the phase-field simulation for $\alpha_{0}=40^{\circ}$, which also gives a small elimination angle. It shows that the mesoscopic model is able to describe the GB evolution for high misorientations if the elimination occurs in the steady regime (i.e., after the initial transient) and the elimination angle is small. In the extreme case of $\alpha_{0}=45^{\circ}$, where the convergent or divergent nature of the GBs is not defined any more, the differences are particularly striking. While the phase-field model predicts a very fast elimination of the misoriented grain, both grains coexist in the mesoscopic simulation and no competition can be detected. The disparity is not surprising since the structure of the phasefield grains is degenerate, lacking clearly defined primary tips, which cannot be correctly described by the mesoscopic model. Such degenerate structures grow at higher tip undercooling than dendrites [44] and can thus leave more place for rebranching from the well-oriented grain. The mesoscopic model can also predict lateral drift of dendrite tips within the dendritic array. Such spacing adjustments can be observed in the the well oriented dendrite in Fig. 12. They are most pronounced at large misorientations $\left(\alpha_{0}>30^{\circ}\right)$.

It seems that the reason for the failure of the mesoscopic model to predict grain competition at misorientations above $30^{\circ}$ is a too scarce generation of new branches from the welloriented grain. In the spatio-temporal plots for $\alpha_{0}=30^{\circ}-45^{\circ}$ in Fig. 12 we can see that no new branches are generated form the red grain. A possible reason is that the envelope is too "stiff" and too difficult to destabilize. In Section 3.3 we have seen that the stagnant-film thickness has a large influence on the stability of the envelope. With a smaller stagnant-film thickness the destabilization of the envelope to 
new branches is made easier. An investigation of the influence of the stagnant-film thickness on the rebranching and the grain competition is therefore made in the next section. A second possible reason for the incorrect predictions is that the generation of new branches is governed by phenomena at a scale that is not described by the mesoscopic model with sufficient accuracy or is not described at all. This could be argued from the findings of Tourret and Karma [44] that show that the branching dynamics at a GB is chaotic and critically depends on the thermal noise around the dendrite tip. It is presently unclear whether noise can play a significant role at the scale at which the mesoscopic envelope description operates and whether it can thus be a missing ingredient in the mesoscopic model.

\subsection{The influence of the stagnant-film thickness}

In Fig. 14, we compare the envelope shapes at $\alpha_{0}=20^{\circ}$, obtained using different values of the stagnant-film thickness, $\delta_{c}$. We can see that a small stagnant-film thickness promotes the destabilization of the envelopes. At $\delta_{c}=0.35 l_{\text {diff }}$, oscillations of the envelope appear at the sides of the dendrite branches. They leave a pronounced trace in the solid-fraction and average-concentration fields inside the envelopes. At $\delta_{c} \geq 0.82 l_{\text {diff }}$ the envelopes of the individual primary arms become smoother. For large stagnant-film thicknesses, $\delta_{c} \geq$ $1.54 l_{\text {diff }}$, the microstructure is not represented in the same way any more. The envelope of the misoriented grain starts to smoothen. Instead of forming new branches at the diverging GB, the envelope expands in a smooth way. While this can be a valid approximation of the dendrite envelope of the columnar zone, the information on the primary spacing of this grain is partly lost.

The stagnant-film thickness also has an influence on the predicted mesoscopic texture, as we can see in Fig. 15. For $\delta_{c} \leq 0.82 l_{\text {diff, }}$, only rebranching from the misoriented grain occurs at the diverging GB, as shown in Fig. 14. These new tertiary branches repeatedly win the competition and block rebranching of the well oriented grain. Both grains survive side by side and the angle of the diverging GB is zero, as can be seen in Fig. 15. With $\delta_{c}=1.18 l_{\text {diff }}$ both the welloriented and the misoriented grain form tertiary branches at the diverging GB. As in the sequence shown in Fig. 14, the well-oriented grain is successful at each branching event and gradually eliminates the misoriented grain. These events are periodic and the GB is thus straight. With large stagnant-film thicknesses, $\delta_{c} \geq 1.54 l_{\text {diff }}$, the rebranching of both grains is less clearly distinguishable. It is seen in the form of an expanding envelope without distinguishable branches (Fig. 14, right). Still, a competition of the expanding envelopes takes place at the diverging GB. In both cases shown in Fig. 15 the misoriented grain is eliminated. The elimination angle is however much smaller. This holds for all moderate misorientation angles, as shown in Fig. 13, where the grain elimination angle, $\left(\theta_{\mathrm{D}}-\theta_{\mathrm{C}}\right)$, across the full range of crystalline misorientations, $\alpha_{0}$, is represented for $\delta_{c}=1.54 l_{\text {diff }}$. Note that for $\delta_{c}<1.18 l_{\text {diff }}$, the grain elimination angle is zero.
On the one hand, we have shown in Section 3.3 that the spacing selection dynamics is most accurate with stagnantfilm thicknesses that are slightly smaller than $l_{\text {diff }}$. On the other hand, accurate predictions of growth competition requires that $\delta_{c} \sim l_{\text {diff. }}$. As a compromise, the latter should be a good calibration of this parameter. $l_{\text {diff }}$ corresponds roughly to the distance at which the solute diffusion field in front of the primary tip is not modified anymore by the curvature of the interface at the tip scale. In the mesocopic model this distance allows a proper matching of the analytical microscopic tip solution to the numerical mesoscopic fields.

\section{Conclusions and perspectives}

We have demonstrated the applicability of the mesoscopic envelope model to columnar dendritic growth in directional solidification. By comparisons to phase-field computations, used as a reference, we have shown that with a proper choice of stagnant-film thickness in the steady state the mesoscopic model correctly reproduces the stable primary spacing, accurately captures the solute diffusion at the scale of the primary spacing and the solutal undercooling ahead of the columnar tips, and accurately predicts the solidification path in the mushy zone behind the columnar front.

We have further shown that the mesoscopic model reproduces spacing adjustments both by tertiary rebranching and by elimination of primary branches. Branching and elimination are also critical phenomena at the origin of growth competition between differently oriented grains. We have demonstrated that the mesoscopic model can reproduce these phenomena and thus the macroscale growth competition for small and moderate misorientation angles, i.e., up to $30^{\circ}$.

Nevertheless, a careful calibration of the stagnant-film thickness has to be performed to obtain reliable predictions. The stagnant-film thickness should be of the order of the diffusion length: $\delta_{c} \sim l_{\text {diff }}=D_{1} / V_{\text {tip }}$. This is particularly important for predicting growth competition, governed by the highly chaotic processes at the grain boundaries. In the case of a single grain growth the simulations are less sensitive to the stagnant-film thickness.

Further investigation is required to fully understand the mechanism of the destabilization of the envelope and to determine the role of the stagnant-film thickness in the destabilization. It also remains to be clarified within which range of solidification parameters steady-state tip laws can provide quantitative predictions of the transients in columnar growth of interacting branches.

Due to the smaller computational cost than for phase-field models, mesoscopic simulations can be performed on a scale that corresponds to the Representative Elementary Volume (REV) used in deriving volume-averaged macroscopic models of solidification processes. In this way, we expect to be able to use mesoscopic simulations as an input for scale bridging to macroscopic models $[30,52]$, thus obtaining a whole simulation chain for multiscale modeling of solidification with 
$\delta_{c}=0.35 l_{\mathrm{diff}}$

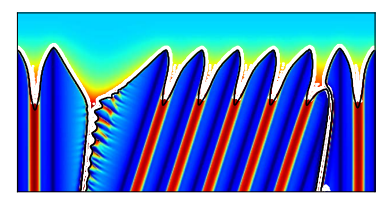

$13 \mathrm{~s}$

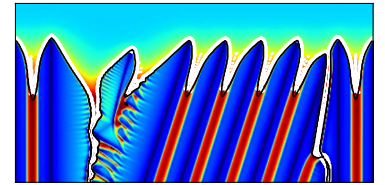

$17 \mathrm{~s}$

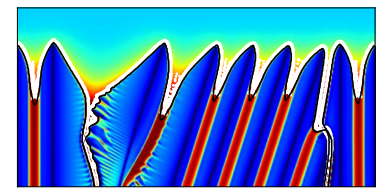

$21 \mathrm{~s}$ $\delta_{c}=0.82 l_{\mathrm{diff}}$

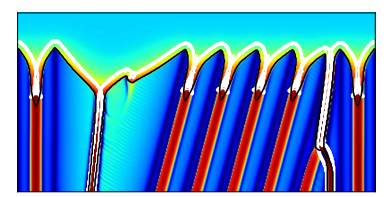

$19 \mathrm{~s}$

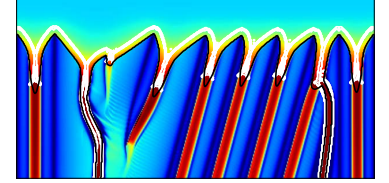

$23 \mathrm{~s}$

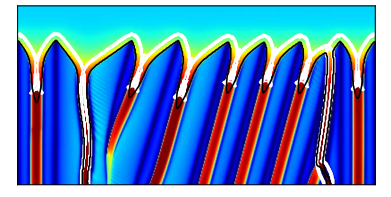

$27 \mathrm{~s}$ $\delta_{c}=1.18 l_{\mathrm{diff}}$

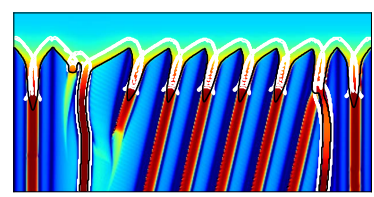

$19 \mathrm{~s}$

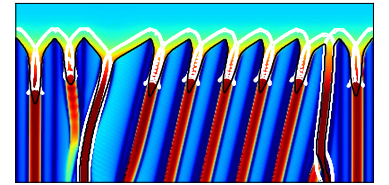

$23 \mathrm{~s}$

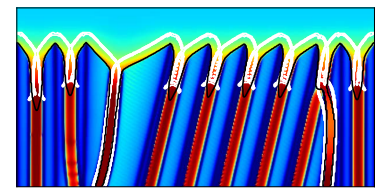

$27 \mathrm{~s}$ $\delta_{c}=1.54 l_{\mathrm{diff}}$

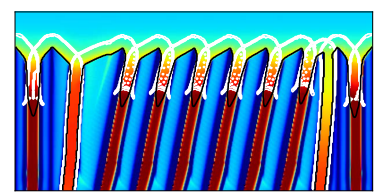

$10 \mathrm{~s}$

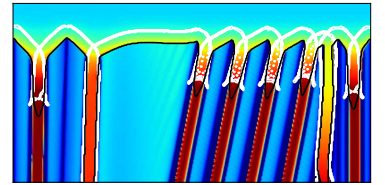

$38 \mathrm{~s}$

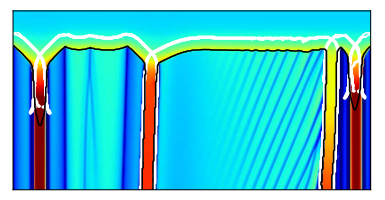

$136 \mathrm{~s}$

Fig. 14. Interactions at the grain boundaries for different stagnant-film thicknesses, $\delta_{c}$.

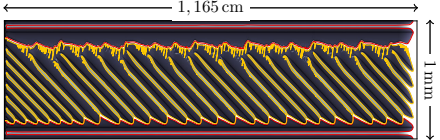

$\delta_{c}=0.35 l_{\text {diff }}$

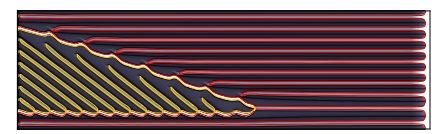

$\delta_{c}=1.18 l_{\text {diff }}$

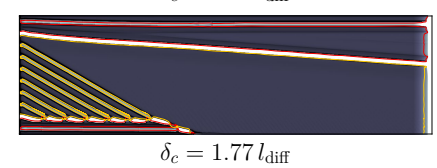

$\delta_{c}=1.77 l_{\text {diff }}$

Fig. 15. The influence of the stagnant-film thickness, $\delta_{c}$, on the growth competition between the well oriented (red contour) and the misoriented (yellow contour) grains $\left(\alpha_{0}=20^{\circ}, t=200 \mathrm{~s}\right.$, solid fraction is superimposed). Note that the horizontal length is displayed compressed by a factor of 0.3 .

reliable accuracy. Other extensions of this work are 3D computations of columnar growth and the inclusion of fluid flow. Both can be achieved with reasonable computational time.

\section{Acknowledgements}

A.V. and M.A. gratefully acknowledge the support to this research by the German Federal Ministry of Research through the German Space Agency DLR under Contract FKZ 50WM1143. M.Z., Y.S. and H.C. gratefully acknowledge the support to this research by the French State through the program "Investment in the future" operated by the National Research Agency (ANR) and referenced by ANR-11 LABX-000801 (LabEx DAMAS).

\section{References}

[1] MICRESS version 6.1, www.micress.de, 2014.

[2] OpenFOAM, version 2.2.2, www.openfoam.org, 2014.

[3] B Böttger, M Apel, B Santillana, and D G Eskin. "relationship between solidification microsctructure and hot cracknig susceptibility for continuous casting of low carbon and high strengh low alloyed steels : A phase-field study". Metallurgical and Materials Transactions A, 44A(8):3765-3777, 2013.

[4] D. J. Browne and J. D. Hunt. a Fixed Grid Front-Tracking Model of the Growth of a Columnar Front and an Equiaxed Grain During Solidification of an Alloy. Numerical Heat Transfer, Part B: Fundamentals, 45(5):395-419, 2004.

[5] B Cantor and A Vogel. Dendritic solidification and fluid flow. Journal of Crystal Growth, 41(1):109-123, nov 1977.

[6] Pierre Delaleau, Christoph Beckermann, Ragnvald H Mathiesen, and Lars Arnberg. Mesoscopic Simulation of Dendritic Growth Observed in X-ray Video Microscopy During Directional Solidification of AlCu Alloys. ISIJ International, 50(12):1886-1894, jan 2010.

[7] G. L. Ding, W. D. Huang, X. Huang, X. Lin, and Y. H. Zhou. On primary dendritic spacing during unidirectional solidification. Acta Materialia, 44(9):3705-3709, 1996.

[8] H B Dong and Peter D Lee. Simulation of the columnar-to-equiaxed transition in directionally solidified Al-Cu alloys. Acta Materialia, 53:659-668, 2005.

[9] J Eiken. Numerical solution of the phase-field equatoin with minized discretization error. IOP Conf Series : Materials Science and Engineering, 33:012105, 2012.

[10] J Eiken, M Apel, V T Witusiewicz, J Zollinger, and U Hecht. Interplay between $\alpha(\mathrm{Ti})$ nucleation and growth during peritectic solidification investigated by phase-field simulations. Journal of Physics: Condensed Matter, 21(46):464104, nov 2009.

[11] J. Eiken, B. Böttger, and I. Steinbach. Multiphase-field approach for multicomponent alloys with extrapolation scheme for numerical application. Physical Review E, 73(6):066122, jun 2006.

[12] Charles-André Gandin, To Carozzani, H Digonnet, S Chen, and Gildas Guillemot. Direct modeling of structures and segregations up to industrial casting scales. JOM, 65(9):1122-1130, 2013.

[13] Charles-André Gandin, M. Eshelman, and Rohit Trivedi. Orientation dependence of primary dendrite spacing. Metallurgical and Materials Transactions A, 27(9):2727-2739, sep 1996.

[14] Z Guo and S M Xiong. Study of dendritic growth and coarsening using a 3-D phase field model: Implementation of the Para-AMR algorithm. 
IOP Conference Series: Materials Science and Engineering, 84:012067, 2015.

[15] S. H. Han and Rohit Trivedi. Primary spacing selection in directionally solidified alloys. Acta Metallurgica et Materialia, 42(1):25-41, jan 1994.

[16] J. D. Hunt. Solidification and Casting of Metals. The Metal Society, London, 1979.

[17] G P Ivantsov. Temperature field around a spherical, cylindrical and needle-like crystal growing in a supercooled melt. Doklady Akademii Nauk SSSR, 58(4):567-569, 1947.

[18] Abdellah Kharicha, M Stefan-Kharicha, Andreas Ludwig, and Menghuai Wu. A scale adaptive dendritic envelope model of solidification at mesoscopic scales. IOP Conference Series: Materials Science and Engineering, 84:012032, 2015.

[19] W. Kurz and D. J. Fisher. Dendrite growth at the limit of stability: tip radius and spacing. Acta Metallurgica, 29(1):11-20, 1981.

[20] W Kurz, B Giovanola, and R Trivedi. Acta Metall., 34:823, 1986.

[21] Wilfried Kurz and David J Fisher. Fundamentals of Solidification. Trans Tech Publications, Aedermannsdorf, Switzerland, 1998.

[22] Junjie Li, Zhijun Wang, Yaqin Wang, and Jincheng Wang. Phase-field study of competitive dendritic growth of converging grains during directional solidification. Acta Materialia, 60(4):1478-1493, 2012.

[23] X. Lin, W. Huang, J. Feng, T. Li, and Y. Zhou. History-dependent selection of primary cellular/dendritic spacing during unidirectional solidification in aluminum alloys. Acta Materialia, 47(11):3271-3280, 1999.

[24] W Losert, B Q Shi, H Z Cummins, and James A Warren. Spatial PeriodDoubling Instability of Dendritic Arrays in Directional Solidification. Physical Review Letters, 77(5):889-891, 1996.

[25] Shu Zu Lu and J. D. Hunt. A numerical analysis of dendritic and cellular array growth: the spacing adjustment mechanisms. Journal of Crystal Growth, 123(1-2):17-34, 1992.

[26] D. Ma and P.R. Sahm. Solidification behavior of superalloy in 939 in melt spinning. Acta Metallurgica et Materialia, 40(2):251-257, feb 1992.

[27] Shaun McFadden and D. J Browne. A front-tracking model to predict solidification macrostructures and columnar to equiaxed transitions in alloy castings. Applied Mathematical Modelling, 33(3):1397-1416, 2009.

[28] Michel Rappaz and Charles-André Gandin. Probabilistic modelling of microstructure formation in solidification processes. Acta Metallurgica et Materialia, 41(2):345-360, 1993.

[29] S Sakane, Tomohiro Takaki, Munekazu Ohno, Takashi Shimokawabe, and Takayuki Aoki. GPU-accelerated 3D phase-field simulations of dendrite competitive growth during directional solidification of binary alloy. IOP Conference Series: Materials Science and Engineering, 84:012063, jun 2015.

[30] Youssef Souhar, Valerio Francesco De Felice, Christoph Beckermann, Hervé Combeau, and Miha Založnik. Three-dimensional mesoscopic modeling of equiaxed dendritic solidification of a binary alloy. Computational Materials Science, 112:304-317, feb 2016.

[31] Ingo Steinbach. Effect of interface anisotropy on spacing selection in constrained dendrite growth. Acta Materialia, 56(18):4965-4971, oct 2008.

[32] Ingo Steinbach, Christoph Beckermann, B Kauerauf, Q Li, and J Guo. Three-dimensional modeling of equiaxed dendritic growth on a mesoscopic scale. Acta Materialia, 47(3):971-982, feb 1999.

[33] Ingo Steinbach, Hermann-Josef Diepers, and Christoph Beckermann. Transient growth and interaction of equiaxed dendrites. Journal of Crystal Growth, 275(3-4):624-638, mar 2005.

[34] Ingo Steinbach, F Pezzolla, Britta Nestler, M. Seeßelberg, R. Prieler, G.J. Schmitz, and J.L.L. Rezende. A phase field concept for multiphase systems. Physica D, 94(3):135-147, jul 1996.

[35] Sonja Steinbach. Einfluss von Strömungen auf die Entwicklung des Mikrogefüges bei der gerichteten Erstarrung von Al-Si und Al-Si-Mg Legierungen. PhD thesis, RWTH Aachen, 2005.

[36] Ying Sun and Christoph Beckermann. Sharp interface tracking using the phase-field equation. Journal of Computational Physics, 220(2):626-653, jan 2007.

[37] Ying Sun and Christoph Beckermann. A two-phase diffuse-interface model for HeleShaw flows with large property contrasts. Physica D:
Nonlinear Phenomena, 237(23):3089-3098, dec 2008

[38] Satyam Suwas and Ranjit Kumar Ray. Crystallographic Texture of Materials. Springer, London, 2014.

[39] Tomohiro Takaki, Munekazu Ohno, Yasushi Shibuta, Shinji Sakane, Takashi Shimokawabe, and Takayuki Aoki. Two-dimensional phasefield study of competitive grain growth during directional solidification of polycrystalline binary alloy. Journal of Crystal Growth, 442:14 24, 2016.

[40] Tomohiro Takaki, Munekazu Ohno, Takashi Shimokawabe, and Takayuki Aoki. Two-dimensional phase-field simulations of dendrite competitive growth during the directional solidification of a binary alloy bicrystal. Acta Materialia, 81:272-283, 2014.

[41] Tomohiro Takaki, Takashi Shimokawabe, Munekazu Ohno, Akinori Yamanaka, and Takayuki Aoki. Unexpected selection of growing dendrites by very-large-scale phase-field simulation. Journal of Crystal Growth, 382:21-25, 2013.

[42] Damien Tourret, Amy J. Clarke, Seth D. Imhoff, Paul J. Gibbs, John W. Gibbs, and Alain Karma. Three-Dimensional Multiscale Modeling of Dendritic Spacing Selection During Al-Si Directional Solidification. JOM, 67(8):1776-1785, 2015.

[43] Damien Tourret and Alain Karma. Multiscale dendritic needle network model of alloy solidification. Acta Materialia, 61(17):6474-6491, oct 2013.

[44] Damien Tourret and Alain Karma. Growth competition of columnar dendritic grains: A phase-field study. Acta Materialia, 82:64-83, jan 2015.

[45] Rohit Trivedi. Interdendritic Spacing: Part II. A Comparison of Theory and Experiment. Metallurgical Transactions A, 15(6):977-982, jun 1984.

[46] A. Wagner, B. A. Shollock, and M. McLean. Grain structure development in directional solidification of nickel-base superalloys. Materials Science and Engineering A, 374(1-2):270-279, 2004.

[47] W Wang, Peter D Lee, and M McLean. A model of solidification microstructures in nickel-based superalloys: Predicting primary dendrite spacing selection. Acta Materialia, 51:2971-2987, 2003.

[48] James A Warren and J S Langer. Stability of dendritic arrays. Physical Review A, 42(6):3518-3525, 1990.

[49] James A Warren and J S Langer. Prediction of dendritic spacings in a directional-solidification experiment. Physical Review E, 47(4):27022713, 1993.

[50] Huang Weidong, Geng Xingguo, and Zhou Yaohe. Primary spacing selection of constrained dendritic growth. Journal of Crystal Growth, 134(1-2):105-115, 1993.

[51] Honglei Yu, Junjie Li, Xin Lin, Lilin Wang, and Weidong Huang. Anomalous overgrowth of converging dendrites during directional solidification. Journal of Crystal Growth, 402:210-214, 2014.

[52] Miha Založnik, Youssef Souhar, Christoph Beckermann, and Hervé Combeau. Upscaling from Mesoscopic to Macroscopic Solidification Models by Volume Averaging. In Frontiers of Solidification Science, TMS Annual Meeting 2016, pages 59-64. TMS, 2016.

[53] Y. Z. Zhou, A. Volek, and N. R. Green. Mechanism of competitive grain growth in directional solidification of a nickel-base superalloy. Acta Materialia, 56(11):2631-2637, 2008. 